\title{
The influence of the sidereal cosmic-ray anisotropies originated on the tail- and nose-boundaries of the heliomagnetosphere (HMS) upon the solar cosmic-ray anisotropy produced inside the HMS
}

\author{
K. Nagashima ${ }^{1}$, I. Kondo ${ }^{2}$, I. Morishita ${ }^{3}$, and R. M. Jacklyn ${ }^{4}$
}

\author{
${ }^{1}$ Solar-Terrestrial Environment Laboratory, Nagoya University, Chikusaku, Nagoya 404-8601, Japan \\ ${ }^{2}$ Institute for Cosmic Ray Research, The University of Tokyo, 5-1-5 Kashinoha, Kashiwa 277-8582, Japan \\ ${ }^{3}$ Dept. of Information Management, Asahi University, 1851 Hozumi, Mizuho, Gifu 501-0296, Japan \\ ${ }^{4}$ Antarctic Division, Department of Science and Technology, Kingston, Tasmania, Australia
}

(Received January 7, 2009; Revised January 30, 2010; Accepted February 26, 2010; Online published August 6, 2010)

\begin{abstract}
The solar diurnal variation of cosmic rays was considered to be fully explained by the diffusion-convection theory. Recently, however, it has been found that the geographic latitude distribution of the yearly averaged diurnal variations observed with the neutron monitors and the muon telescopes on the ground does not agree with that expected from the theory. The difference between the two distributions is observed almost every year, and it is especially remarkable in the solar activity minimum period in the positive polarity state of the solar polar magnetic field, when the diurnal variation reduces its magnitude and shifts its phase towards the morning side. It is shown that such a difference is produced by the seasonal variation of the sidereal heliotail-in and helionose-in anisotropies with respective right ascensions of $6 \mathrm{~h}$ and $18 \mathrm{~h}$ according to the following process. Generally, if any sidereal anisotropy from the right ascension $\alpha$ is subject to the seasonal variation with its maximum and minimum at the times when the Earth is closest to and farthest from the source of the anisotropy, respectively, located through its direction at the boundary of the HMS, it produces a yearly averaged residual flux from $0 \mathrm{~h}$ LT in solar geographic polar coordinates regardless of its direction $(\alpha)$. This residual flux from $0 \mathrm{~h}$ LT produces the difference mentioned above.

Key words: Cosmic rays (CR), solar and sidereal anisotropies of CR, solar modulation of CR, seasonal variation of CR sidereal anisotropy, neutron monitors, polarity of solar magnetic field, heliomagnetosphere (HMS), CR acceleration on HMS boundary, major axis of HMS.
\end{abstract}

\section{Introduction}

The modulation of galactic cosmic rays in the heliomagnetosphere (HMS) has been considered to be described by the diffusion-convection theory first proposed by Parker (1958) and theoretically formulated by Gleeson and Axford (1967). On the basis of this theory, many authors have numerically solved the so-called diffusion-convection equation and tried to explain various modulation phenomena (e.g. Gleeson, 1969; Fisk, 1971; Dorman and Milovinova, 1973; Kota, 1975; Moraal and Gleeson, 1975; Earl, 1976; Jokipii and Kopriva, 1979; Moraal et al., 1979; Jokipii and Davila, 1981; Bieber and Pomerantz, 1983).

The solar diurnal variation of cosmic rays was also one of the subjects of the modulation. The most remarkable feature of the variation is seen in its phase shift from the evening side to the morning side with the two solar-cycles' periodicity corresponding to the polarity reversal of the solar polar magnetic field from the negative $(\mathrm{N}-)$ to the positive (P-) state, as will be shown later (cf. Nagashima et al., 1986).

Copyright (C) The Society of Geomagnetism and Earth, Planetary and Space Sciences (SGEPSS); The Seismological Society of Japan; The Volcanological Society of Japan; The Geodetic Society of Japan; The Japanese Society for Planetary Sciences; TERRAPUB.

doi:10.5047/eps.2010.02.012
The theoretical simulation of the variation with a special emphasis on the effect of the polarity-dependent heliolatitudinal drift motion of cosmic rays that have been pointed out by Jokipii and Kopriva (1979) could explain the observed shift and showed that the diurnal variation is fully explained by the diffusion-convection theory (Munakata and Nagashima, 1986). Recently, however, this conclusion has been contra-indicated by the following evidence. As stated in Abstract, the observed geographic latitude distribution of the yearly averaged diurnal variations largely deviates from that derived from the solar anisotropy $\left(\mathbf{F}_{\mathrm{DC}}\right)$ due to the diffusion-convection of cosmic rays with any reasonable rigidity spectrum. It will be shown in the following sections that such a difference would come from the seasonal variation of the sidereal heliotail-in anisotropy $\left(\mathbf{F}_{\mathrm{T}}\right)$ of cosmic rays from a right ascension $\left(\alpha_{\mathrm{T}}\right)$ of $6 \mathrm{~h}$ and the declination $\left(\delta_{\mathrm{T}}\right)$ of $-24^{\circ}$ and also of the helionose-in anisotropy $\left(\mathbf{F}_{\mathrm{H}}\right)$ from $\alpha_{\mathrm{H}}=18 \mathrm{~h}$ and $\delta_{\mathrm{H}}>0^{\circ}$, both of which are thought to be produced respectively by the cosmic-ray accelerations on the tail and nose boundaries of the HMS (cf. Nagashima et al., 1998, 2005).

Generally, any sidereal anisotropy $\mathbf{F}_{\mathrm{U}}$ of an origin $\mathrm{U}$ with the right ascension $\alpha_{\mathrm{U}}$ interacts all the time with the solar anisotropy $\mathbf{F}_{\mathrm{DC}}$ due to the diffusion-convection of cosmic rays in the HMS. However, if $\mathbf{F}_{\mathrm{U}}$ is constant for 1 year, 

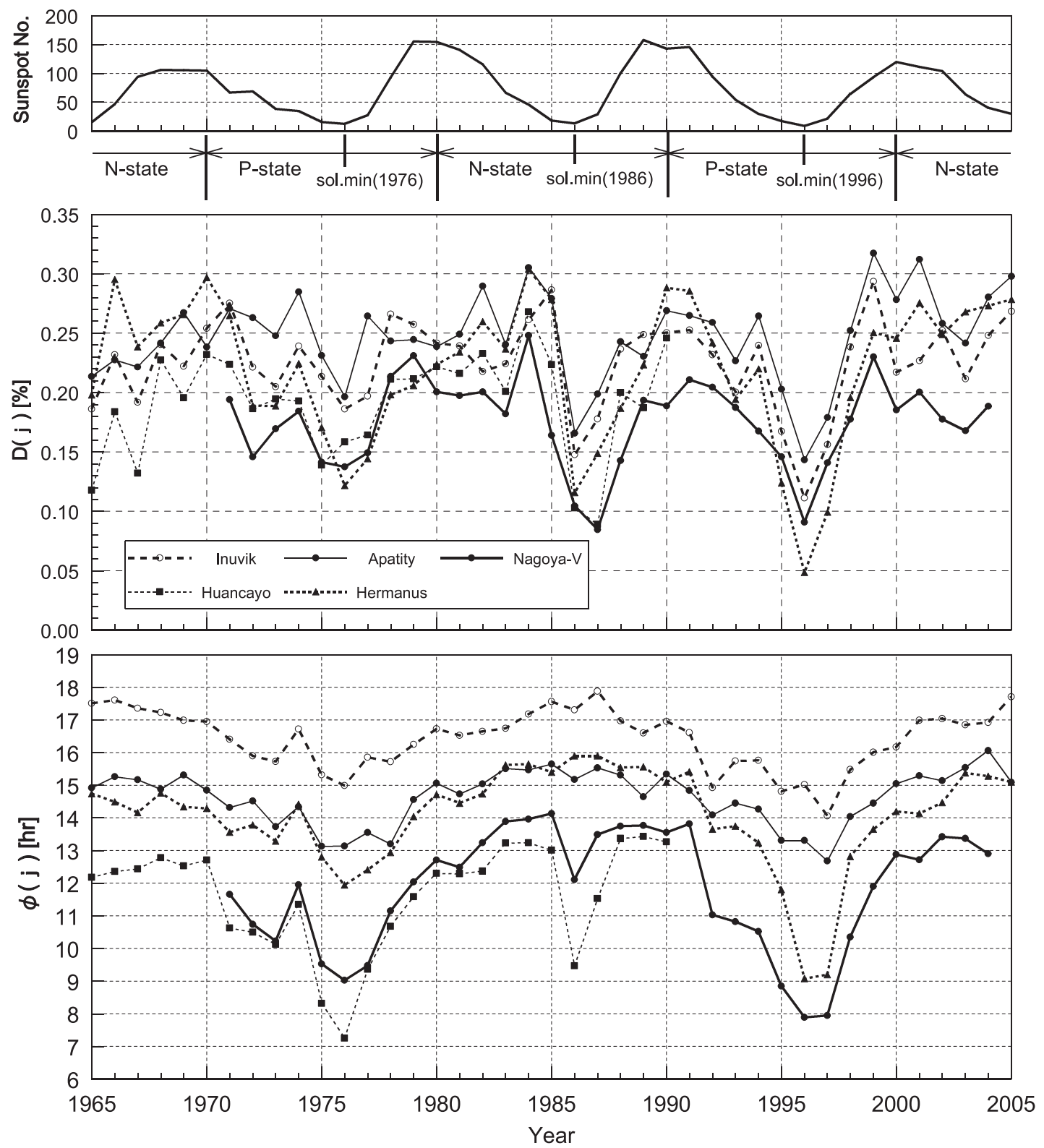

Fig. 1. Long-term variation of the amplitude $\mathrm{D}(j)$ and phase $\phi(j)$ of the solar diurnal variation $\mathbf{D}(j)$ of the neutrons and the vertical muon component observed at $j$ stations, Inuvik $\left(\lambda=68^{\circ} \mathrm{N}, \phi=134^{\circ} \mathrm{W} ; p_{\mathrm{c}} \sim 0.2 \mathrm{GV}\right)$, Apatity $\left(68^{\circ} \mathrm{N}, 33^{\circ} \mathrm{E} ; 0.7 \mathrm{GV}\right)$, Hermanus $\left(34^{\circ} \mathrm{S}, 19^{\circ} \mathrm{E} ; 4.9 \mathrm{GV}\right)$, Huancayo $\left(12^{\circ} \mathrm{S}, 75^{\circ} \mathrm{W} ; 13.0 \mathrm{GV}\right)$ and Nagoya $(\mathrm{V})\left(35^{\circ} \mathrm{N}, 137^{\circ} \mathrm{E} ; 11.5 \mathrm{GV}\right)$. $\mathrm{P}$ and $\mathrm{N}$ : the positive and negative states of the solar polar magnetic field.

the yearly average of $\mathbf{F}_{\mathrm{U}}$ in the geographic polar coordinate system $(r, \theta, \phi)$ does not affect the yearly average of $\mathbf{F}_{\mathrm{DC}}$ because $\mathbf{F}_{\mathrm{U}}$ rotates anticlockwise with a constant angular velocity in solar time, and the yearly average of its $\phi$-dependent term becomes zero. On the contrary, if $\mathbf{F}_{\mathrm{U}}$ would be subject to a seasonal variation with its maximum and minimum at the times when the Earth is closest to and farthest from the source of the anisotropy, respectively, located through its direction at the boundary of the HMS, it would produce a yearly averaged residual flux $\mathbf{F}_{\text {USO }}$ from $0 \mathrm{~h} \mathrm{LT}$ in the solar geographic coordinate regardless of its direction $\left(\alpha_{\mathrm{U}}\right)$. $\mathbf{F}_{\mathrm{USO}}$ produces the solar diurnal variation $\mathbf{D}(j)_{\text {USO }}$ with a phase $\phi(j)_{\text {USO }}$ at a $j$-station on the Earth. $\mathbf{D}(j)_{\text {USO }}$ interacts with the solar diurnal variation $\mathbf{D}(j)_{\mathrm{DC}}$ with a phase $\phi(j)_{\mathrm{DC}}$ due to $\mathbf{F}_{\mathrm{DC}}$ and produces a resultant vector $\mathbf{D}(j)_{\mathrm{UD}}\left(=\mathbf{D}(j)_{\mathrm{USO}}+\mathbf{D}(j)_{\mathrm{DC}}\right)$ with a phase
$\phi(j)_{\mathrm{UD}}$. The relative position between $\mathbf{D}(j)_{\mathrm{UD}}$ and $\mathbf{D}(j)_{\mathrm{DC}}$ on the solar first harmonic coordinates $(X, Y)$ is classified into the following three types depending on the difference between $\phi(j)_{\text {USO }}$ and $\phi(j)_{\mathrm{DC}}$, on the premise that $\mathbf{D}(j)_{\mathrm{USO}}$ and $\mathbf{D}(j)_{\mathrm{DC}}$ are in the fourth quadrant $\left(270^{\circ}-360^{\circ}\right)$ and the second to third quadrants $\left(90^{\circ}-270^{\circ}\right)$ of the coordinates, respectively, as

$$
\begin{aligned}
& \phi(j)_{\mathrm{UD}}-\phi(j)_{\mathrm{DC}}\left\{\begin{array}{l}
>0 \\
=0 \\
<0
\end{array} \text { or } \pi\right. \\
& \text { for } \phi(j)_{\mathrm{USO}}-\phi(j)_{\mathrm{DC}}\left\{\begin{array}{c}
< \\
= \\
>
\end{array}\right\} \pi .
\end{aligned}
$$



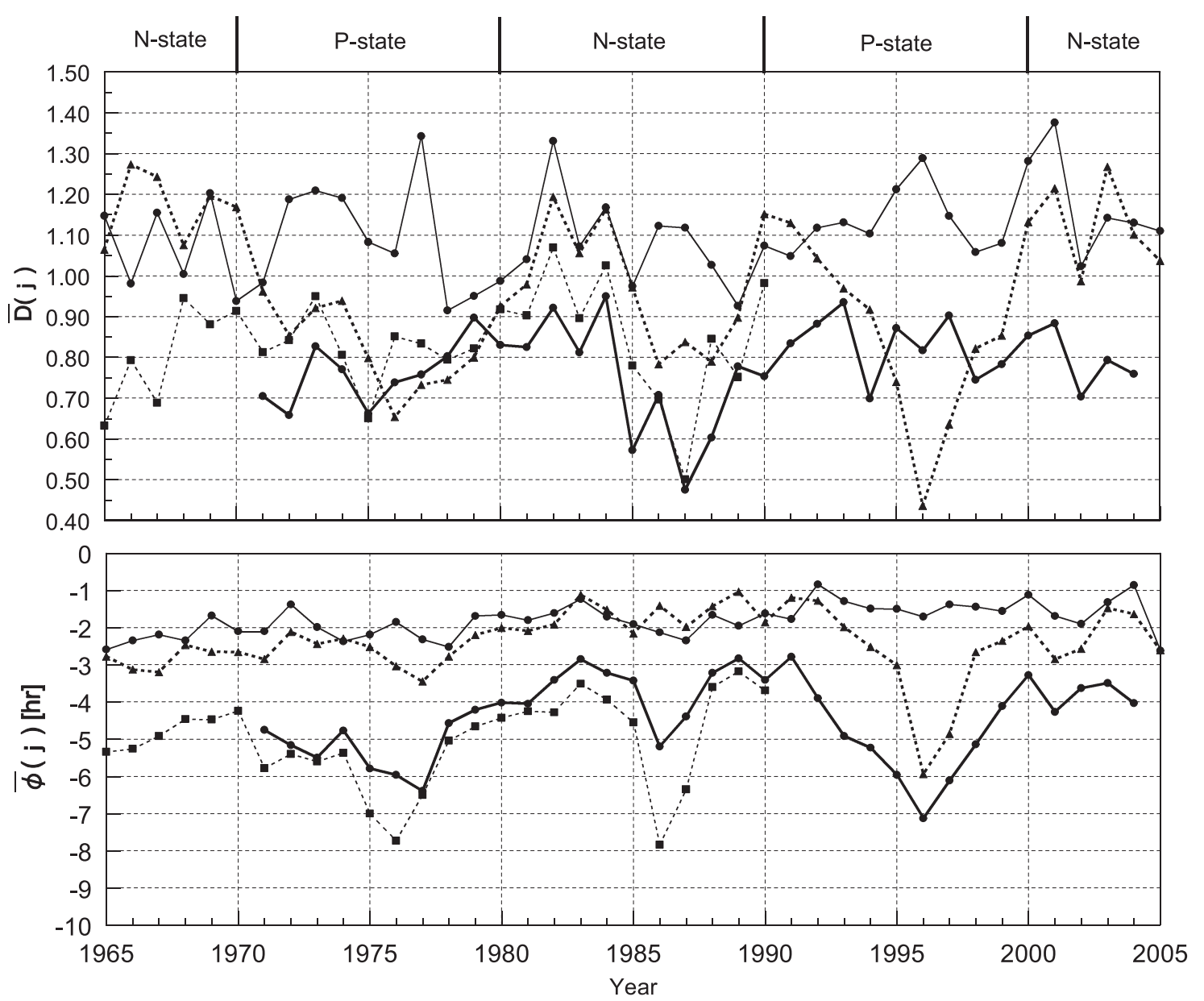

Fig. 2. Long-term variation of the amplitude $\overline{\mathrm{D}}(j)$ and phase $\bar{\phi}(j)$ of the normalized solar diurnal variation $\overline{\mathbf{D}}(j)$ at the stations in Fig. 1 (cf. Eq. (5)).

The deviation of $\phi(j)_{\mathrm{UD}}$ from $\phi(j)_{\mathrm{DC}}$ of the three types would produce the difference of the observed variation from that derived from the diffusion-convection theory.

In 1998, the existence of such a sidereal anisotropy called the tail-in anisotropy $\mathbf{F}_{\mathrm{T}}\left(\alpha_{\mathrm{T}}=6 \mathrm{~h}\right)$ and of its seasonal variation was discovered by observations at Hobart underground muon station (geographic lat. $(\lambda) 43^{\circ} \mathrm{S}$, long. $(\varphi) 147^{\circ} \mathrm{E}$; median energy $\left(E_{\mathrm{m}}\right) 184 \mathrm{GeV}$; Jacklyn, 1966, 1986; Nagashima et al., 1995, 1998). However, its residual flux $\mathbf{F}_{\mathrm{TSO}}$ in the geographic coordinates was not explicitly found by observations because of the large influence of $\mathbf{F}_{\mathrm{DC}}$. The existence of $\mathbf{F}_{\mathrm{TSO}}$ is confirmed in the present analysis and provides a firm basis for the interpretation of the abovementioned difference between the distributions of the yearly averaged diurnal variations observed and those theoretically derived by the diffusion-convection of cosmic rays.

\section{Solar Diurnal Variation of Cosmic Rays}

The solar anisotropy $\mathbf{F}(y)$ of cosmic rays with an amplitude $\mathrm{F}(y)$ and a phase $\Phi(y)$ at a time $y(0-1)$ in a unit of 1 year produces the diurnal variation $\mathbf{D}(j, y)$ with an amplitude $\mathrm{D}(j, y)$ and a phase $\phi(j, y)$ at a station $j$. For the convenience for the following discussion, $y=0$ is set at the time of the December solstice. The yearly averages of these variables are expressed without $y$. The observed $\mathbf{D}(j, y) \mathrm{s}$ used in the following analysis are those of neutron monitors $\left(E_{\mathrm{m}} \sim 20 \mathrm{GeV}\right)$ at the old and new WDC-C2 Centers for Cosmic Rays ${ }^{* 1}$ and the muon telescopes at Nagoya $\left(E_{\mathrm{m}} \sim 60 \mathrm{GeV}, \lambda=35.1^{\circ} \mathrm{N}, \varphi=137^{\circ} \mathrm{E}\right.$; Sekido et al. 1975; Fujii et al., 2000) in the period of 1965 2007.

$\mathbf{D}(j, y)$ is subject to solar modulation caused by the variation of solar activity. The most remarkable systematic modulation is the long-term variation of $\phi(j)$ with the twosolar-cycle periodicity (cf. Nagashima et al., 1986). Some examples of the variation are shown in Fig. 1. In the P-state, $\phi(j)$ shifts toward the morning side, while in the N-state, it stays near the evening side $(\sim 18 \mathrm{~h})$. Such a phenomenon is called the polarity dependence of the variation. To study the dependence, it is necessary to derive $\mathbf{F}(y)$ every year from the observation of $\mathbf{D}(j, y)$ 's at many stations on the assumption that $\mathbf{F}(y)$ has a specific structure in space, such as the one proposed by the diffusion-convection theory. Such a study is too troublesome to be performed every year for a long-term period and, furthermore, it derives inevitably a solution in the framework of the assumed model no matter how large the error is. To avoid such a troublesome problem, the following simple method for the analysis is pro-

${ }^{* 1}$ The Cosmic-Ray Section of the Institute of the Physical and Chemical Research, Itabashi, Tokyo and Solar-Terrestrial Environment Laboratory, Nagoya University. 

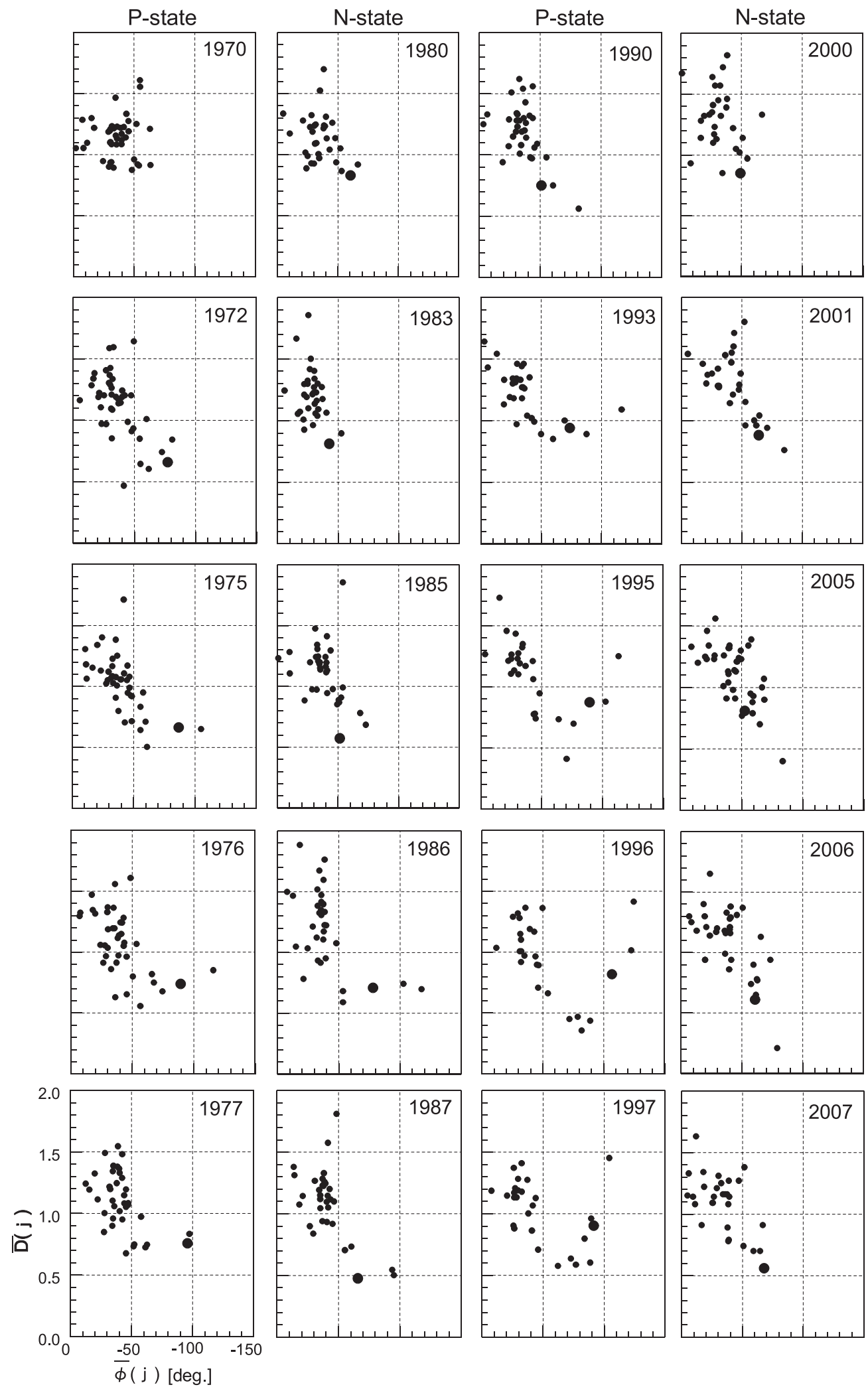

Fig. 3. Distributions of $\overline{\mathbf{D}}(j)$ 's on the $\overline{\mathrm{D}}-\bar{\phi}$-diagrams in the respective selected years during the period of 1970-2007, showing their dependence on the solar activity and the polarity of the solar polar magnetic field. The bold point is for the vertical (V) muon telescope at Nagoya $\left(35^{\circ} \mathrm{N}\right)$.

posed.

$\mathbf{F}(y)$ contains two unknown factors: one is its phase (i.e., the direction in space) $\Phi(y)$, and the other is its structure (i.e., the space distribution and the rigidity spectrum). The observed $\mathbf{D}(j, y)$ is related to these factors by the following relations.

$$
\begin{aligned}
& \mathrm{D}(j, y)=\zeta(j, y) \mathrm{F}(y), \\
& \phi(j, y)=\Phi(y)+\Delta \phi_{\mathrm{g}}(j, y),
\end{aligned}
$$

where $\zeta(j, y)$ and $\Delta \phi_{\mathrm{g}}(j, y)$ are the coupling coefficients 


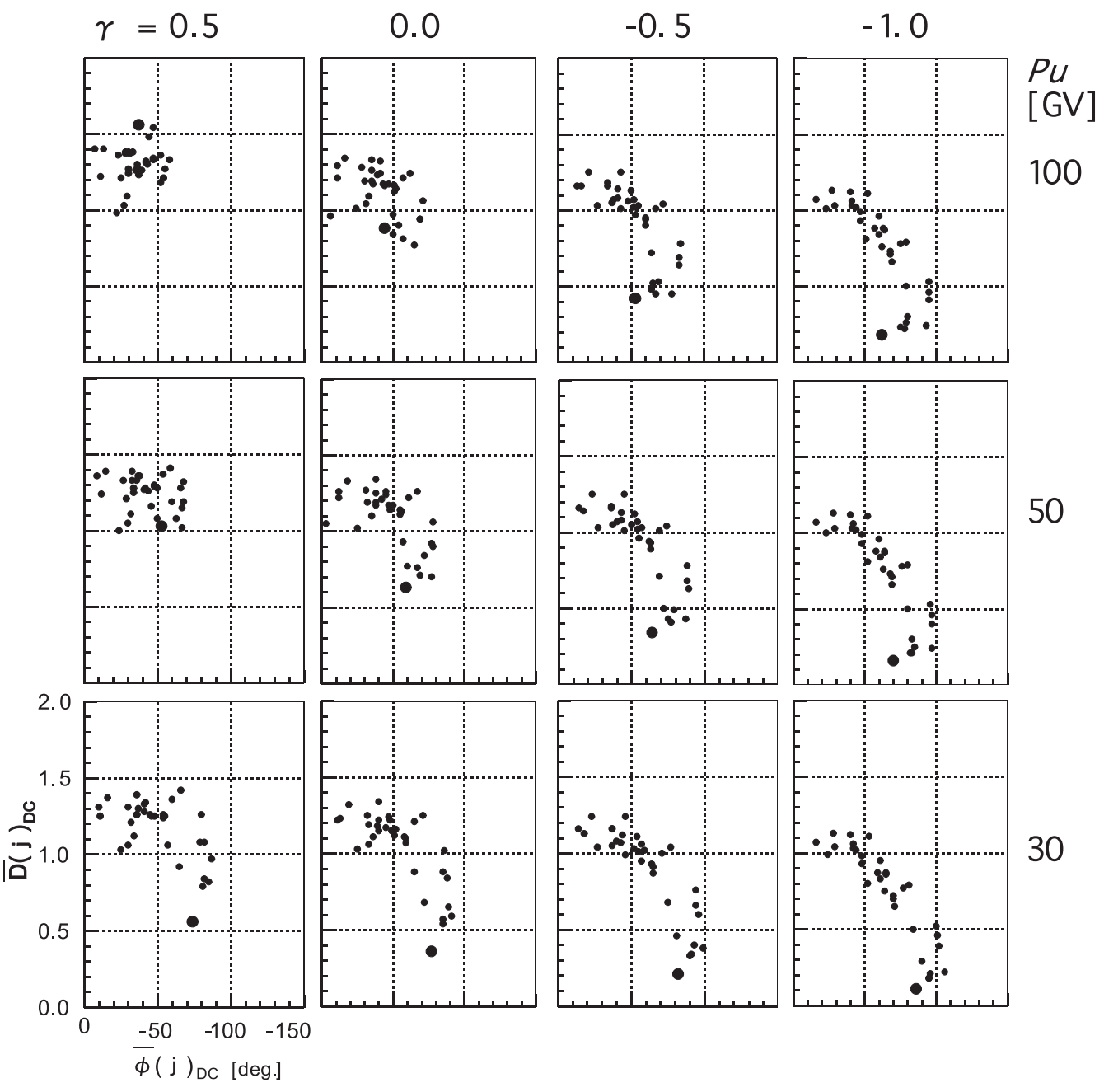

Fig. 4. $\overline{\mathrm{D}}-\bar{\phi}$-diagrams showing the distributions of the theoretical $\overline{\mathbf{D}}(j)_{\mathrm{DC}}$ 's due to the diffusion-convection of cosmic rays for various combinations of parameters $\gamma$ and $p_{\mathrm{u}}$ of the rigidity spectrum. The stations in these figures are not always the same as those in Fig. 3. The bold point is for the vertical (V) muon telescope at Nagoya $\left(35^{\circ} \mathrm{N}\right)$.

determined by the geomagnetic deflection and the atmospheric attenuation of cosmic rays, depending on the structure of $\mathbf{F}(y)$, (Yasue et al., 1982; Inoue et al., 1983). Hereafter, the coefficient $\Delta \phi_{\mathrm{g}}(j, y)$ is called the geomagnetic deflection angle for simplicity. If the unknown factor $\Phi(y)$ could be eliminated from Eq. (2), the structure of $\mathbf{F}(y)$ could be more easily studied, for instance, even by the analysis of $\mathbf{D}(j, y)$ at a single station instead of the complicate mathematical analysis as above. The elimination is possible by the following approximation method. Among the neutron monitor stations, there are high-latitude stations where the geographic longitude of the cosmic-ray asymptotic orbital direction in the geomagnetosphere is insensitive to the cosmic-ray rigidity. In other words, the variation of $\phi(j, y)$ in Eq. (2) at these stations expresses the variation of $\Phi(y)$ and can be used for the elimination of $\Phi(y)$ in Eq. (2) at other stations. From among these stations, we have chosen Inuvik $\left(\lambda=68.4^{\circ} \mathrm{N}, \varphi=226.3^{\circ} \mathrm{E}\right.$; geomagnetic cut-off rigidity $p_{\mathrm{c}}=0.16 \mathrm{GV}$ ) for this purpose, as it has been in constant operation for a long-term period and can observe a considerably large amplitude $\mathrm{D}$ (INU, $y$ ) with good accuracy owing to the wide spread of the latitude distribution of the asymptotic orbits toward low latitudes with the decrease of the rigidity, despite its high latitude location. In this case, $\Phi(y)$ in Eq. (2) is expressed as

$$
\begin{aligned}
\Phi(y) & =\phi(\mathrm{INU}, y)-\Delta \phi_{\mathrm{g}}(\mathrm{INU}, y) \\
& =\phi(\mathrm{INU}, y)-\Delta \phi_{\mathrm{g}},
\end{aligned}
$$

where $\Delta \phi_{\mathrm{g}}$ is $-9^{\circ} \pm 1^{\circ}$ for any kind of the rigidity spectrum (cf. Yasue et al., 1982). If we take the difference $\Delta \phi(j, y)$ between $\phi$ (INU, $y)$ and $\phi(j, y)$, we can eliminate $\Phi(y)$ as follows

$$
\begin{aligned}
\Delta \phi(j, y) & =\phi(j, y)-\phi(\mathrm{INU}, y) \\
& =\Delta \phi_{\mathrm{g}}(j, y)-\Delta \phi_{\mathrm{g}} .
\end{aligned}
$$

By using $\Delta \phi(j, y)$ and $\mathrm{D}(j, y)$, we introduce a normalized $\overline{\mathbf{D}}(j, y)$ which is independent of $\Phi(y)$ as

$$
\begin{aligned}
& \overline{\mathrm{D}}(j, y)=\mathrm{D}(j, y) / \mathrm{D}(\mathrm{INU}, y), \\
& \bar{\phi}(j, y)=\Delta \phi(j, y) .
\end{aligned}
$$

In this way, we can compare $\overline{\mathbf{D}}(j, y)$ 's in different periods for the study of the time variation of the structure of $\mathbf{F}(y)$. The $\mathbf{D}(j)$ s shown in Fig. 1 are transformed into $\overline{\mathbf{D}}(j)$ 's in Fig. 2. The variation of $\bar{\phi}(j)$ in high latitudes is very small, 
indicating that the polarity dependence of $\phi(j)$ in these regions is mainly due to the variation of $\Phi$ of the anisotropy. On the other hand, the polarity dependence of $\bar{\phi}(j)$ remarkable in low latitudes would be due to the variation of the rigidity spectrum of the anisotropy. In the following analysis, $\overline{\mathbf{D}}(j, y)$ is shown on a graph called a $\overline{\mathrm{D}}-\bar{\phi}$-diagram whose vertical and horizontal axes express $\overline{\mathrm{D}}$ and $\bar{\phi}$, respectively. The distributions of $\overline{\mathbf{D}}(j)$ 's for the respective years, being extracted from those in the period of 19652007 , are shown on the $\overline{\mathrm{D}}-\bar{\phi}$-diagrams in Fig. 3 in order to show their long-term variations. Some $\overline{\mathbf{D}}(j)$ 's are excluded from the diagram because of their unreasonably large deviation from the majority of distribution. Corresponding to these observations, the variation $\overline{\mathbf{D}}(j)_{\mathrm{DC}}$ due to the solar anisotropy $\mathbf{F}_{\mathrm{DC}}$ of the diffusion-convection theory is obtained on the assumption that the rigidity spectrum $f(p) d p$ of $\mathbf{F}_{\mathrm{DC}}$ is given by

$$
\begin{aligned}
f(p) d p & =f_{\mathrm{o}} \cdot p^{\gamma} d p & & \text { for } p \leq p_{\mathrm{u}}, \\
& =0 & & \text { for } p>p_{\mathrm{u}},
\end{aligned}
$$

in which the exponent $\gamma$ and the upper cut-off rigidity $p_{\mathrm{u}}$ are parameters (cf. Yasue et al., 1982; Fujimoto et al., 1984). The distributions of $\overline{\mathbf{D}}(j)_{\mathrm{DC}}$ 's at selected stations for various combinations of $\gamma$ and $p_{\mathrm{u}}$ are shown on the respective $\overline{\mathrm{D}}-\bar{\phi}$-diagrams in Fig. 4 . It is noted that the selected stations do not always coincide with those in Fig. 3, and that these distributions do not show any similarity with the observations in Fig. 3. The characteristic difference between the theoretical and the observed distributions appears in the location of the center of curvature of the distribution curve; it is left-downwards in the former case and right-upward in the latter. The most remarkable dissimilarity of the distribution of $\overline{\mathbf{D}}(j)$ from the theory was observed in the solar activity minimum period (1996) in Fig. 3. This distribution has a very short radius of curvature. Such a distribution was observed in the adjacent years of 1996 and appeared also in the P-state of the 1970s.

The disagreement between $\overline{\mathbf{D}}(j)$ and $\overline{\mathbf{D}}(j)_{\mathrm{DC}}$ can be demonstrated more quantitatively on the $\overline{\mathrm{D}}-\bar{\phi}$-diagram by the following method. We plot on the diagram all the $\overline{\mathbf{D}}(j)_{\mathrm{DC}}$ 's at a $j$-station obtained for various combination of $\gamma$ and $p_{\mathrm{u}}$ and draw the iso- $\gamma$ and the iso- $p_{\mathrm{u}}$ lines, respectively, through the points with the corresponding $\gamma$ and $p_{\mathrm{u}}$ values in order to constitute the $\gamma-p_{\mathrm{u}}$ net. If $\overline{\mathbf{D}}(j)$ is outside the net area, it can not be explained solely by the anisotropy with the assumed spectrum. Two kinds of $\overline{\mathbf{D}}(j)$ distribution which deviate from the net area are shown in Fig. 5 in which the numbers attached to some points express the observation years in the 1970s and 1990s, respectively, in the P-state. Most of the marked points at Nagoya are on the right-outside of the net area and can not be explained by the anisotropy unless $\gamma$ is very large and $p_{\mathrm{u}}$ becomes very small so that the diurnal variation at Nagoya can be produced by a rigidity spectrum with an extremely narrow region between the geomagnetic cut-off rigidity $\left(p_{\mathrm{c}}=11.5 \mathrm{GV}\right)$ and $p_{\mathrm{u}}$ (for example $\sim 15 \mathrm{GV}$ ). However, such a spectrum with the large $\gamma$ can not explain other $\overline{\mathbf{D}}(j)$ 's in high latitudes. The abnormally rightward deviation of $\overline{\mathbf{D}}(j)$ from the respective net area in the P-state was also observed at Huancayo $\left(\lambda=12.0^{\circ} \mathrm{S}, p_{\mathrm{c}}=13.0 \mathrm{GV}\right)$, Tokyo $\left(35.8^{\circ} \mathrm{N} ; 11.5 \mathrm{GV}\right)$, and Beijing $\left(40.1^{\circ} \mathrm{N} ; 8.9 \mathrm{GV}\right)$, which are characterized by the large $\left|\Delta \phi_{\mathrm{g}}(j, y)\right|$ defined in Eq. (2) (cf. Yasue et al., 1982). On the contrary, the marked points at Hermanus on the left diagram in Fig. 5 do not show such an abnormality, although they do show another difficulty, as follows. As can be seen in the figure, almost all of the $\overline{\mathbf{D}}$ (Hermanus)'s are in the left-outside region of the net area regardless of the mark. Such a deviation from the net area cannot be attributed to the observation error of the direction $\Phi$ derived from $\phi(\mathrm{INU})$, as almost all of the deviations are one-sided with respect to the iso- $p_{\mathrm{u}}$ line of $10^{3} \mathrm{GV}$. It has been found that this region is forbidden not only for $\overline{\mathbf{D}}$ (Hermanus)'s with the present spectral form but also for those with any reasonable spectra as far as the anisotropy is unidirectional. Hereafter, such differences between $\overline{\mathbf{D}}(j)$ and $\overline{\mathbf{D}}(j)_{\mathrm{DC}}$ in Figs. 3, 4 and 5 are called the D-anomaly for simplicity. It will be made clear later in this article that the two types of the D-anomaly in Fig. 5 correspond respectively to the two of three types of the deviation of $\phi(j)_{\mathrm{UD}}$ from $\phi(j)_{\mathrm{DC}}$ defined in Eq. (1).

Finally, a decisive piece of evidence has been found that cannot be explained within the framework of the diffusionconvection theory and strikes at the root of the two kinds of the D-anomaly mentioned above. This evidence was found in the data of the diurnal variation in the period from late 1995 to early 1996 in the P-state when solar activity was at a minimum. In this period, some $\mathbf{D}(j, y)$ 's, mainly in low and middle latitudes, reversed or changed their phase $\phi(j, y)$ 's from the daytime side $(6 \mathrm{~h}-12 \mathrm{~h}-18 \mathrm{~h})$ toward the nocturnal side ( $18 \mathrm{~h}-0 \mathrm{~h}-6 \mathrm{~h})$, while the others reduced their magnitudes. Similar events were also observed in the solar activity minimum period of 1977 in the P-state. Hereafter, these phenomena are referred to as the phase anomaly as a whole, in contrast to the D-anomaly. Before showing the phase anomaly, it would be reasonable to present, as the background of the anomaly, the general trend of the solar cycle dependence of $\mathbf{D}(j, y)$ in the P-state by means of the following consecutive movements of $\mathbf{D}\left(j, y_{i}\right)$ averaged over every solar rotation $\left(y_{i}\right)$, which are composed of a series of $\sum_{i=1}^{i_{\mathrm{f}}} \mathbf{D}\left(j, y_{i}\right)$ for $i_{\mathrm{f}}=1,2,3 \cdots$, being plotted in the solar first harmonic coordinates $(X-Y)$ and connected with neighboring points by the lines. Hereafter, such a representation of $\mathbf{D}\left(j, y_{i}\right)$ 's is called the accumulative $\mathbf{D}\left(j, y_{i}\right)$ or simply $\Sigma \mathbf{D}(j, y)$. Some $\Sigma \mathbf{D}(j, y)$ 's at several stations in 1989-2000 are shown in Fig. 6. The movements of $\mathbf{D}(j, y)$ 's are smooth and mutually harmonious with each other, showing the gradual phase advancement toward the morning side characteristic of the P-state in the period of 1990-1997, except the one at Beijing station, which shows the persistent, systematic and large annual variations superposed on the general trend common to the variations at other stations. This additional phenomenon will be identified later as a form of the phase anomaly after the phase reversals in the minimum period (1995-1996) mentioned above. The phase anomaly has been fully analyzed. Occurrences of the phase anomaly are designated by the arrows in Fig. 6. Some examples of the phase anomaly in this minimum period are shown by the enlarged $\Sigma \mathbf{D}(j, y)$ 's in Fig. 7. In the figure, $\mathbf{D}(j, y)$ 's with the bold-faced symbol and with the mark A in the winter season of 1995-1996 ex- 

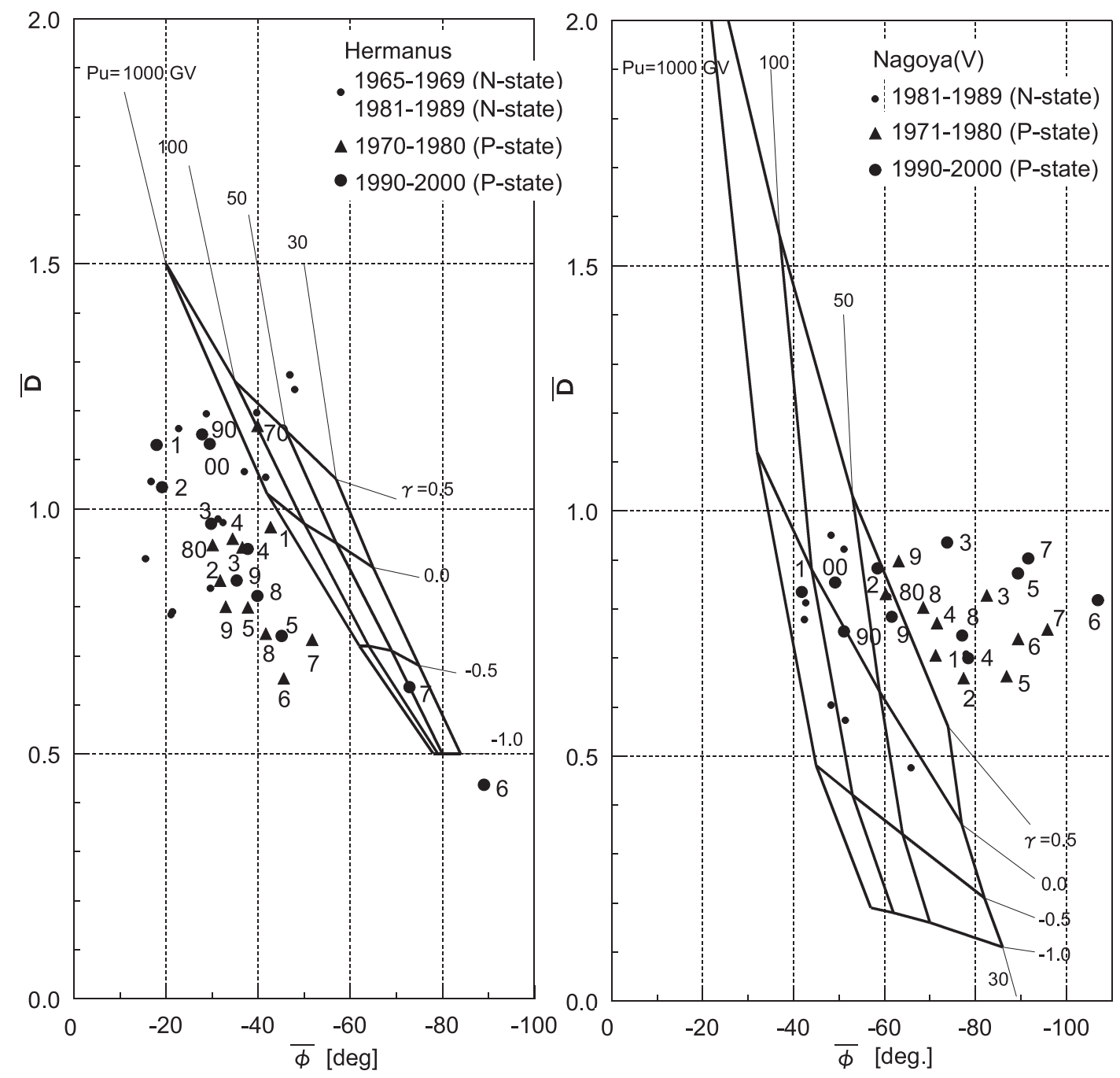

Fig. 5. $\overline{\mathrm{D}}-\bar{\phi}$-diagram showing the distributions of $\overline{\mathbf{D}}(\mathrm{Herm})$ at Hermanus $\left(34^{\circ} \mathrm{S}, 19^{\circ} \mathrm{E} ; 4.9 \mathrm{GV}\right)$ during the period (1965-2000) on the left and $\overline{\mathbf{D}}(\mathrm{V})$ at Nagoya $\left(35^{\circ} \mathrm{N}, 137^{\circ} \mathrm{E} ; 11.5 \mathrm{GV}\right)$ during the period $(1971-2000)$ on the right. $\overline{\mathbf{D}}(j)$ in the net area can be explained by the solar anisotropy with the parameter $\gamma$ and $p_{\mathrm{u}}$ at the point. The number attached each point expresses the observation year in the 1970s and 1990s in the P-state.

press the phase anomaly, and those with the mark B in this season of the following year seem to show the persistency of the anomaly although they are not so clear. Note that the bold-faced period includes the precursory period (Oct., Nov., and Dec.) of the phase reversal (Jan., Feb., and Mar.) and is called hereafter the anomaly-enhanced period (AEP). The characteristics of the anomaly with the mark A are as follows:

(1) $\mathbf{D}(j, y)$ reverses its direction toward $18 \mathrm{~h} \sim 24 \mathrm{~h}$ from $\sim 12 \mathrm{~h} \sim 18 \mathrm{~h}$ LT at stations with high latitude-like phase displacements, while it rotates its direction anticlockwise toward $0 \mathrm{~h} \sim 6 \mathrm{~h}$ from $\sim 6 \mathrm{~h} \sim 12 \mathrm{~h}$ at stations with low latitude-like phase displacements (cf. Fig. 7). For the sake of convenience, these two types of variation are called the loop-type and the morningtype, respectively. Almost all the phase shifts toward the nocturnal side occurred in January of 1996, and its duration period of the reversal depends on $\lambda$ and the cut-off rigidity $p_{\mathrm{c}}$ at the station; it is less than several solar rotations.
(2) The magnitude $R(j)$ of the total sum of $\mathbf{D}(j, y)$ 's in the respective duration periods in Fig. 8 shows a northsouth (N-S) asymmetric latitude distribution which has the maximum at Hermanus $\left(\lambda=34.4^{\circ} \mathrm{S}\right)$, a considerably large value even at the South Pole $\left(\lambda=90^{\circ} \mathrm{S}\right)$, and almost no response at the northern high-latitude stations Inuvik $\left(\lambda=68.4^{\circ} \mathrm{N}\right)$, Thule $\left(\lambda=76.6^{\circ} \mathrm{N}\right)$, and so forth. Note that the exceptionally large $R(j)$ observed at Climax $\left(\lambda=39.4^{\circ} \mathrm{N}\right)$, Rome $\left(\lambda=41.9^{\circ} \mathrm{N}\right)$, and Jungfraujoch $\left(\lambda=46.6^{\circ} \mathrm{N}\right)$ in the northern hemisphere in Fig. 8 seems not to be contradictory to the $\mathrm{N}-\mathrm{S}$ asymmetry, as will be explained later, and also that $R(j)$ 's of the morning-type variation at Tokyo $\left(\lambda=35.8^{\circ} \mathrm{N}\right)$, Nagoya $\left(\lambda=35.1^{\circ} \mathrm{N}\right)$ and Beijing $\left(\lambda=40.1^{\circ} \mathrm{N}\right)$ are excluded from the figure because of their different character.

(3) The reversed vectors at Hermanus do not overlap with or cross other vectors before or after the reversal, which is contrary to those at other stations, and seem to show the anticlockwise movement with time if the three vectors right before the reversals are included (cf. 


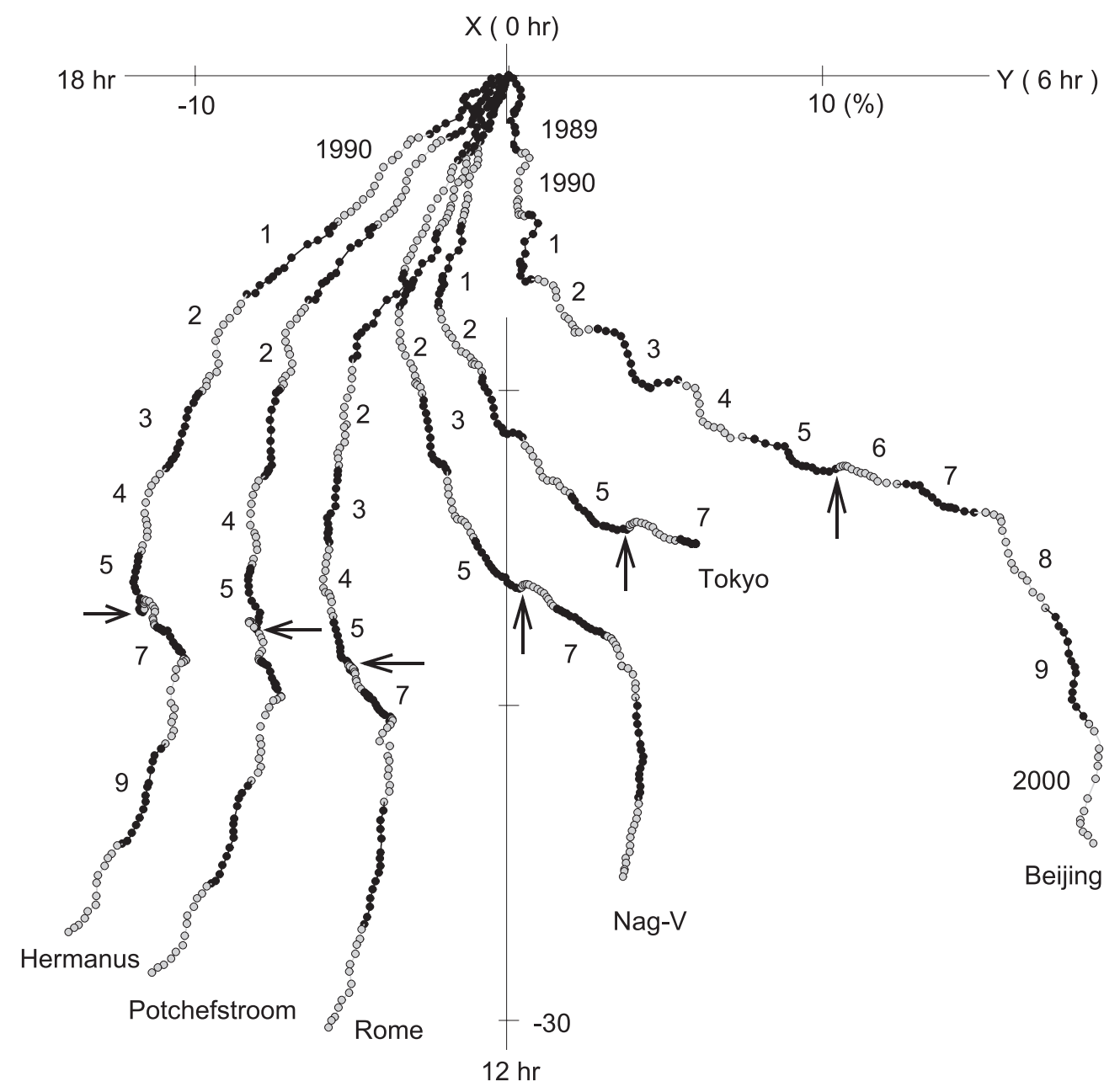

Fig. 6. Solar cycle dependence of $\mathbf{D}\left(j, y_{i}\right)$ 's averaged over every solar rotation $\left(y_{i}\right)$ in the P-state (1989-2000) in terms of the $\Sigma \mathbf{D}\left(j, y_{i}\right)$ representation defined in the text. Beijing $\left(40^{\circ} \mathrm{N}, 8.9 \mathrm{GV}\right)$, Tokyo $\left(36^{\circ} \mathrm{N}, 11.5 \mathrm{GV}\right)$, Nagoya $\left(35^{\circ} \mathrm{N}, 11.5 \mathrm{GV}\right)$, Rome $\left(42^{\circ} \mathrm{N}, 6.2 \mathrm{GV}\right)$, Potchefstroom $\left(27^{\circ} \mathrm{S}, 7.0\right.$ $\mathrm{GV})$ and Hermanus $\left(34^{\circ} \mathrm{S}, 4.9 \mathrm{GV}\right)$. The number expresses the year in the $1990 \mathrm{~s}$ and the arrow expresses the period of the occurrence of the phase anomaly in the winter season of 1995-1996.

Fig. 7).

(4) In relation to this movement of $\mathbf{D}(j, y)$ at Hermanus, the precursory phenomena of the phase anomaly seem to have appeared as the amplitude reduction of $\mathbf{D}(j, y)$ at other stations in the several periods of Oct. Nov., and Dec. 1995 (cf. Fig. 7).

First of all, it is clear that the phase shift toward the nocturnal side can not be explained by the diffusion-convection theory in the P-state (Munakata and Nagashima, 1986). Characteristic (3) suggests the influence of some sidereal anisotropy superposed on the $\mathbf{F}(y)_{\mathrm{DC}}$, and characteristic (2) indicates that the anisotropy is a sharply concentrated cosmic-ray flux from some direction near the latitude $(\lambda=$ $34.4^{\circ} \mathrm{S}$ ) of Hermanus. Characteristic (1) indicates that the right ascension $(\alpha)$ of the anisotropy would be in a range between $1 \mathrm{~h}-6 \mathrm{~h}-13 \mathrm{~h}$ and probably near the center $(\alpha \sim 7 \mathrm{~h})$ of the range as the reversed vectors are directed toward the direction in the nocturnal range $(18 \mathrm{~h} \sim 0 \mathrm{~h} \sim 6 \mathrm{~h} \mathrm{LT})$ in January of 1996. As such conditions can be satisfied by the sidereal tail-in anisotropy $\mathbf{F}_{\mathrm{T}}$ from the direction of $\delta_{\mathrm{T}}=-24^{\circ}$ and $\alpha_{\mathrm{T}}=6 \mathrm{~h}$, it would be reasonable to assume that the phase anomaly in Figs. 7 and 8 would be due to this anisotropy. In order to enforce this assumption, the trajectories of the asymptotic orbital latitudes of cosmic rays as a function of rigidity at several stations are plotted in Fig. 9 to show their positions relative to the presumed source direction $\left(24^{\circ} \mathrm{S}\right)$ (cf. Inoue et al., 1983). The trajectories at Potchefstroom $\left(\lambda=26.7^{\circ} \mathrm{S}\right)$, Hermanus $\left(\lambda=34.4^{\circ} \mathrm{S}\right)$, and Kerguelen $\left(\lambda=49.4^{\circ} \mathrm{S}\right)$ with the large $R(j)$ 's are adjacent to the source line $\left(\delta_{\mathrm{T}}=-24^{\circ}\right)$ and, among them, the one at Hermanus with the largest $R(j)$ crosses the line at the rigidity of $\sim 50 \mathrm{GV}$. This seems to indicate that the median rigidity of the anisotropy responsible for the reversal would be $\sim 50 \mathrm{GV}$. The exceptionally large $R(j)$ 's at the three northern stations (Climax, Rome, and Jungfraujoch) in Fig. 8 are almost comparable with that at the southern station (Hermanus). This indicates that the rigidity spectrum of the anisotropy would be of a power type with a negative exponent $(\gamma<0)$ as these $R(j)$ s are produced effectively by the anisotropy in the rigidity region of $9 \sim 15 \mathrm{GV}$ for the three northern stations and of $\sim 50 \mathrm{GV}$ for the southern station where their trajectories show respectively the nearest approach to the center direction of the anisotropy. This spectrum can not produce any significant $R(j)$ at high northern latitude stations as the nearest approach of their 


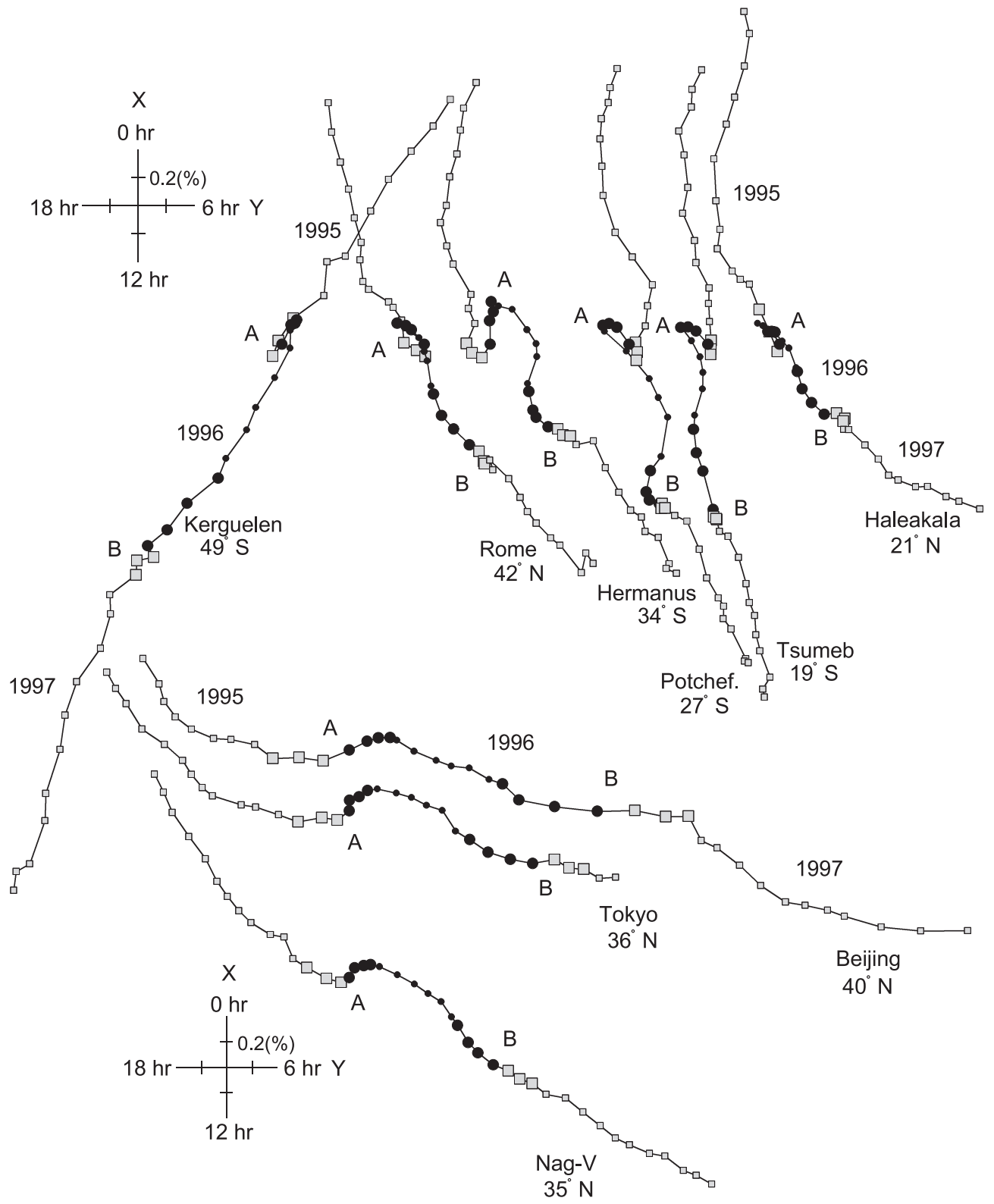

Fig. 7. $\Sigma \mathbf{D}(j, y)$ showing the phase reversals by the bold-faced symbols with the mark A in the winter season of $1995-1996$ and with the B in the same season of the following year. The reversal starts from near January in the case of A. The bold-faced period includes the precursory period (Oct., Nov., and Dec.) of the phase reversal. The scales of the upper six and lower three curves are different from each other. Kerguelen $\left(39^{\circ} \mathrm{S}\right)$, Rome $\left(42^{\circ} \mathrm{N}\right)$, Hermanus $\left(34^{\circ} \mathrm{S}\right)$, Potchefstroom $\left(27^{\circ} \mathrm{S}\right)$, Tsumeb $\left(19^{\circ} \mathrm{S}\right)$, Haleakala $\left(21^{\circ} \mathrm{N}\right)$, Beijing $\left(40^{\circ} \mathrm{N}\right)$, Tokyo $\left(36^{\circ} \mathrm{N}\right), \mathrm{Nagoya}^{\circ}\left(35^{\circ} \mathrm{N}\right)$.

trajectories occurs in the low rigidity region where the influence of the anisotropy on the ground is very small (cf. Fig. 9).

The influence of $\mathbf{F}(y)_{\mathrm{T}}$ also appears in the anomalyenhanced period (AEP) in the next winter season shown by the bold-faced symbols with the mark B in Fig. 7. Although these vectors do not show the clear phase reversal, their movements can be regarded as the phase anomaly as will become clear later. Among them, $\mathbf{D}(j, y)$ 's at Hermanus and Potchefstroom show a morning-type variation in the period from around November that is different from the looptype variation starting from January in the previous season. This difference in type would be due to the difference of $\phi(j, y)_{\mathrm{DC}}$ 's between the two periods, as will be made clear later. In the same period, $\mathbf{D}(j, y)$ at the low-latitude station Beijing also starts its phase change toward $\sim 6 \mathrm{~h} \mathrm{LT}$, and this can not be explained by the diffusion-convection theory. In marked contrast with the clear plateau-type variation at Beijing $\left(\lambda=40.1^{\circ} \mathrm{N} ; p_{\mathrm{c}}=8.9 \mathrm{GV}\right), \mathbf{D}(j, y)$ at Tokyo $\left(\lambda=35.8^{\circ} \mathrm{N} ; p_{\mathrm{c}}=11.5 \mathrm{GV}\right)$ shows only a small change, and the one at Nagoya $\left(\lambda=35.1^{\circ} \mathrm{N}, p_{\mathrm{c}}=11.9 \mathrm{GV}\right)$ does not show any recognizable change. This would be due to the fact that the larger anomaly would be more likely to appear for larger $\left|\Delta \phi_{\mathrm{g}}(j, y)\right|$, as its values at Beijing and Tokyo are larger than that at Nagoya by about $1.5 \sim 2 \mathrm{~h}$ and $\sim 1 \mathrm{~h}$, respectively (cf. Yasue et al., 1982; Fujimoto et al., 1984). The reason for the $\Delta \phi_{\mathrm{g}}(j, y)$-dependence of the anomaly will be made clear later.

In the next section, a detailed explanation will be given for the origin of the phase anomaly, and at the same time it will be shown that this phase anomaly could explain the D-anomaly pointed out previously. 


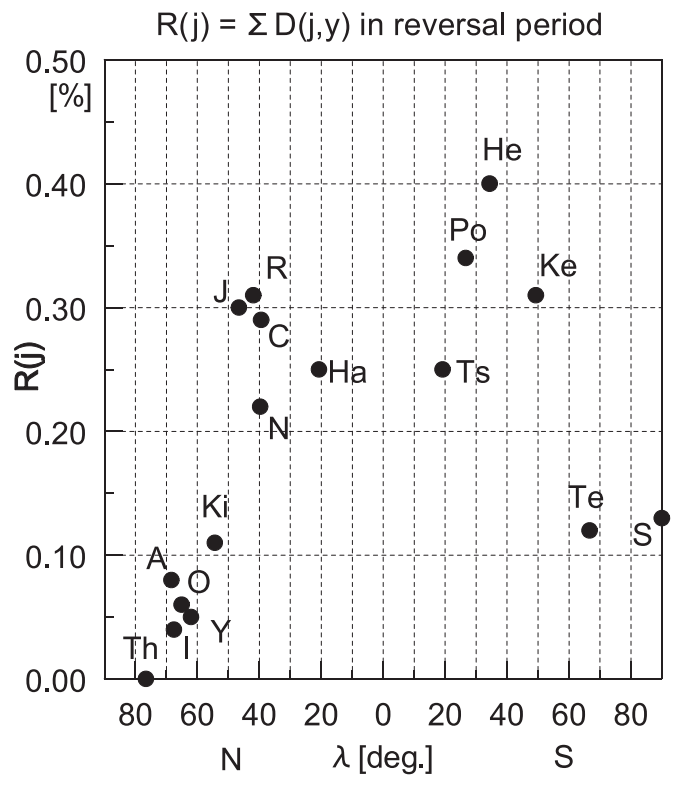

Fig. 8. The latitude distribution of the magnitudes $R(j)$ 's of the total sum of $\mathbf{D}(j, y)$ 's in the respective reversal periods. S: South Pole, Te: Terre Adelie, Ke: Kerguelen, He: Hermanus, P: Potchefstroom, Ts: Tsumeb, Ha: Haleakala, C: Climax, N: Newark, R: Rome, J: Jungfraujoch, Ki: Kiel, Y: Yakutsk, O: Oulu, A: Apatity, I: Inuvik, Th: Thule.

\section{Influence of the Tail-in Anisotropy on the Solar Anisotropy}

The characteristics of the tail-in anisotropy are as follows (cf. Nagashima et al., 1998, 2004, 2005; Nagashima and Fujii, 2006).

(1) The tail-in anisotropy is the sharply concentrated excess flux $\mathbf{F}_{\mathrm{T}}$ with axis-symmetric distribution along the direction of $\alpha=6 \mathrm{~h}$ and $\delta=-24^{\circ}$.

(2) $\mathbf{F}_{\mathrm{T}}$ is thought to be produced by the acceleration of galactic cosmic rays on the tail boundary of the HMS.

(3) $\mathbf{F}_{\mathrm{T}}$ increases and decreases in the P- and N-states, respectively, and varies proportionally to the solar activity.

(4) The magnitude of $\mathbf{F}_{\mathrm{T}}$ at the Earth's orbit is influenced by the strong magnetic field near the Sun and shows its maximum at the December solstice, when the Earth is closest to the tail and its minimum when it is the farthest from the tail, at the June solstice. Therefore, the tail-in anisotropy is expressed by $\mathbf{F}(y)_{\mathrm{T}}$ at a time $y$ $(0-1)$ in 1 year intervals in the sidereal polar coordinate system $(r, \alpha, \delta)$ and also by $\mathbf{F}(y)_{\text {TSo }}$ in the geographic polar coordinate system $(r, \theta, \phi)$.

According to the analysis of Nagashima et al. (1998), $\mathbf{F}(y)_{\mathrm{T}}$ at the June solstice $(y= \pm 0.5)$ was not supposed to be exactly zero, but it is assumed to be zero hereafter to simplify the following analysis. On this assumption, $\mathbf{F}(y)_{\text {TSO }}$ is expressed by

$$
\begin{aligned}
\mathrm{F}(y)_{\mathrm{TSO}} & =\mathrm{F}(0)_{\mathrm{TSO}} \cdot(1+\cos 2 \pi y) / 2, \\
\Phi(y)_{\mathrm{TSO}} & =\Phi(0)_{\mathrm{TSO}}-2 \pi y, \\
\theta(y)_{\mathrm{TSO}} & =\pi / 2-\delta_{\mathrm{T}},
\end{aligned}
$$

in which $y$ is measured from the December solstice. $\mathbf{F}(y)$ TSO can not be expressed on the geographic equato-

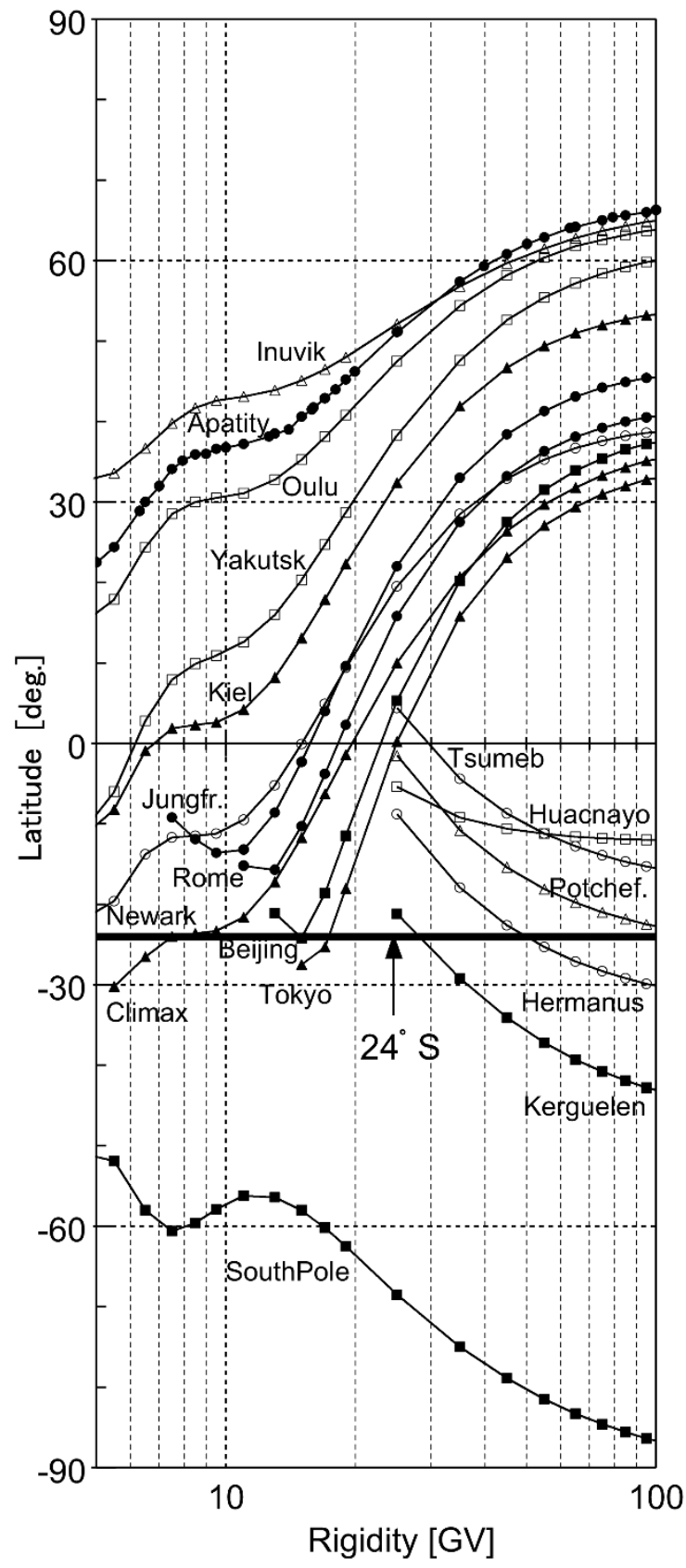

Fig. 9. Rigidity dependence of the geographic latitude of cosmic-ray asymptotic orbital direction in the geomagnetic field at $j$-station. The horizontal thick line $\left(24^{\circ} \mathrm{S}\right)$ expresses the direction $\left(\delta_{\mathrm{T}}=-24^{\circ}\right)$ of the tail-in anisotropy.

rial first harmonic coordinates $(X, Y)$, because its sharply concentrated excess flux from the off-equatorial direction $(\theta \neq \pi / 2)$ is expressed by the series of the spherical harmonic components - at least up to the 2nd order $(n=2)$. The seasonal variation of $\mathrm{F}(y)$ TSO can be visualized indirectly by its product 'the diurnal variation $\mathbf{D}(j, y)_{\mathrm{TSO}}$ ' expressed by

$$
\begin{aligned}
& \mathrm{D}(j, y)_{\mathrm{TSO}}=\zeta(j, y)_{\mathrm{TSO}} \cdot \mathrm{F}(y)_{\mathrm{TSO}}, \\
& \phi(j, y)_{\mathrm{TSO}}=\Phi(y)_{\mathrm{TSO}}+\Delta \phi_{\mathrm{g}}(j, y)_{\mathrm{TSO}},
\end{aligned}
$$

where $\zeta(j, y)_{\text {TSO }}$ and $\Delta \phi_{\mathrm{g}}(j, y)_{\mathrm{TSO}}$ are the coupling coefficients between $\mathbf{D}(j, y)_{\text {TSO }}$ and $\mathbf{F}(y)_{\text {TSO }}$. It is noted that 


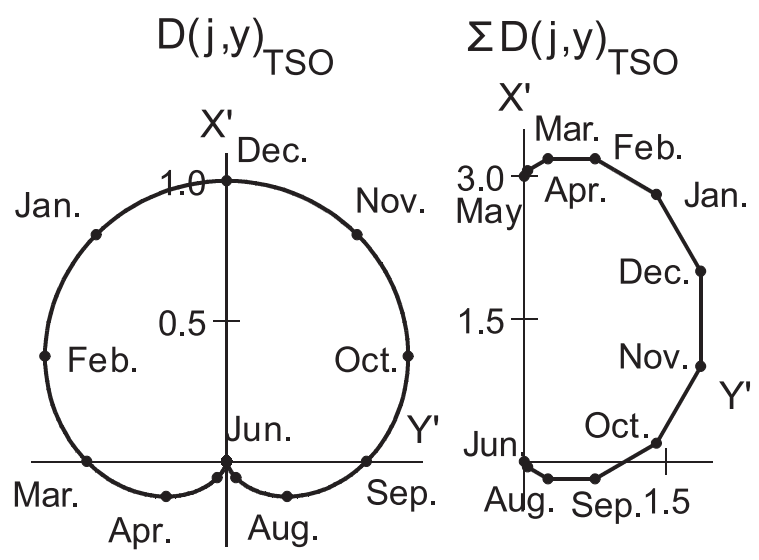

Fig. 10. The seasonal variation of $\mathbf{D}(j, y)_{\text {TSO }}$ in Eq. (8) on the solar harmonic diagram $\left(X^{\prime}, Y^{\prime}\right)$ on the left side and its $\Sigma \mathbf{D}(j, y)_{\text {TSO }}$ on the right. The $X^{\prime}$-axis is in the direction of $\mathbf{D}(j, 0)$ TSO whose magnitude is normalized to unity.

$\mathbf{D}(j, y)_{\text {TSO }}$ is the north-south asymmetric function whose $\Delta \phi_{\mathrm{g}}(j, y)_{\text {TSO }}$ is determined by the rigidity spectra of the $1 \mathrm{st}$ and 2 nd order spherical harmonic components and that it is different from $\Delta \phi_{\mathrm{g}}(j, y)$ in Eq. (2), which is determined by the spectrum of the 1st order spherical harmonic component. The seasonal variation of $\mathbf{D}(j, y)_{\text {TSO }}$ is shown on the new $X^{\prime}-Y^{\prime}$ harmonic coordinates in Fig. 10, whose $X^{\prime}$-axis is in the direction of $\mathbf{D}(j, 0)_{\mathrm{TSO}}$, its magnitude being normalized to unity. Its $\Sigma \mathbf{D}(j, y)_{\text {TSO }}$ is also shown on the right side in Fig. 10. $\mathbf{D}(j, y)_{\text {TSO }}$ modulates $\mathbf{D}(j, y)_{\mathrm{DC}}$ due to the diffusion-convection of cosmic rays into $\mathbf{D}(j, y)_{\mathrm{TD}}$ called the T-modulated variation, as

$$
\mathbf{D}(j, y)_{\mathrm{TD}}=\mathbf{D}(j, y)_{\mathrm{TSO}}+\mathbf{D}(j, y)_{\mathrm{DC}} .
$$

The simulation of $\mathbf{D}(j, y)_{\mathrm{TD}}$ is obtained on the assumption that

$$
\begin{aligned}
& \mathrm{D}(j, y)_{\mathrm{DC}}=1, \\
& \phi(j, y)_{\mathrm{DC}}=\phi_{0}, \\
& \mathrm{D}(j, 0)_{\mathrm{TSO}}=\beta_{0}, \\
& \phi(j, 0)_{\mathrm{TSO}}=0^{\circ},
\end{aligned}
$$

where $\beta_{0}$ and $\phi_{0}$ are parameters. $\Sigma \mathbf{D}(j, y)_{\mathrm{TD}}$ for various combinations of $\beta_{0}$ and $\phi_{0}$ is shown on the $X^{\prime}-Y^{\prime}$ coordinate in Fig. 11 whose $X^{\prime}$-axis is in the direction of $\mathbf{D}(j, 0)_{\text {TSO }}$. They show the three kinds of variation: the loop-type for $\beta_{0}>1$, the morning-type whose phase shifts toward the morning side for $\beta_{0}<1$ and $\phi_{0}<180^{\circ}$ and the eveningtype whose phase shifts toward the evening side for $\beta_{0}<1$ and $\phi_{0}>180^{\circ}$. All of these variations are called the phase anomaly due to $\mathbf{F}(y)$ Tso regardless of their phase reversal toward the nocturnal side. The morning-type anomalies are observed at Beijing, Tokyo, and Nagoya in Fig. 7. It is clear that the $\Delta \phi_{\mathrm{g}}(j, y)$-dependence observed in Fig. 7 is due to the difference of $\phi_{0}$ caused by the difference of $\Delta \phi_{\mathrm{g}}(j, y)$. On the other hand, the evening-type does not appear in this period (1996), but will be observed when $\phi(j, y)_{\mathrm{DC}}$ shifts toward the evening side, as will be shown later. On the contrary, the loop-type is too clear to match the observation in Fig. 7, and its start time of the reversal is clearly different from the observation (January) due to the large vectors
$\mathbf{D}(j, y)_{\text {TSO }}$ 's in the period from July to December. One solution to this difficulty is to assume that $\mathbf{F}(y)_{\text {TSO }}$ was very small in the above period by chance and suddenly increased in January to produce the reversal. This assumption can not necessarily be denied as the acceleration of cosmic rays at the tail boundary of the HMS producing the anisotropy is not always constant through 1 year. Another solution is to modify the distribution of $\mathbf{F}(y)$ Tso on the Earth's orbit. To date, the distribution has been assumed to be axissymmetric with respect to the line through the December and June solstices. This assumption is reasonable for the cosmic rays with high rigidities $(\sim 184 \mathrm{GV})$ observed at Hobart underground station, but the situation would be different for those with low rigidities $(\sim 20 \mathrm{GV})$ observed by the neutron monitors. In such a low rigidity region, the cosmic rays are easily guided by the spiral helio-magnetic field. As a result, $\mathbf{F}_{\mathrm{T}}$ flowing in the left-side region of the meridian plane of $\alpha=6 \mathrm{~h}$ on the sidereal equatorial plane $\left(\delta=0^{\circ}\right)$ of the bird's eye view from the north $\left(\delta=90^{\circ}\right)$ is deflected toward the side-boundary of the HMS and approaches the Earth with difficulty, while the flux in the other side region is guided by the magnetic field and arrives easily at the Earth by changing its direction from $\alpha=6 \mathrm{~h}$ to some late hours. On this assumption, Eq. (7) is tentatively modified as follows,

$$
\begin{gathered}
\mathrm{F}(y)_{\mathrm{TSO}}=\left\{\begin{array}{r}
\mathrm{F}(0)_{\mathrm{TSO}} \cdot(1+\cos 2 \pi y) / 2 \\
\text { for } 0<y<0.5, \\
\mathrm{~F}(0)_{\mathrm{TSO}} \cdot(1+\cos 2 \pi y) / 4 \\
\text { for } 0.5 \leq y \leq 1.0,
\end{array}\right. \\
\Phi(y)_{\mathrm{TSO}}=\left\{\begin{array}{r}
\Phi(0)_{\mathrm{TSO}}-2 \pi(y-1.0 / 24) \\
\text { for } 0<y<0.5, \\
\Phi(0)_{\mathrm{TSO}}-2 \pi y . \\
\text { for } 0.5 \leq y \leq 1.0 .
\end{array}\right.
\end{gathered}
$$

The modified $\Sigma \mathbf{D}(j, y)_{\mathrm{TD}}$ is shown in Fig. 12, which relaxes its loop-type configuration and starts its reversal nearly from January, as required by the observation. This indicates that such a type of modification of $\mathbf{F}(y)_{\mathrm{T}}$ would be reasonable in the low rigidity region $(\sim 20 \mathrm{GeV})$.

The phase anomaly was observed not only in this period (1996) but also in the same winter season (Nov.Mar.) of the solar activity minimum period (1976-1977) in the P-state when $\mathbf{F}(y)_{\mathrm{T}}$ was expected to be enhanced (cf. Nagashima et al., 2005; Nagashima and Fujii, 2006). The observed anomaly in the latter period (1976-1977), however, was not as large as that in the present period (1996), as $\mathbf{F}(y)_{\text {DC }}$ was not as small as that in this period (1996) (cf. Figs. 1 and 2). The phase anomalies can also be observed even in the active period of solar cycle (1992-1994) in the $\mathrm{P}$-state although they are not as clear as those in the previous minimum period (1995-1996). In Fig. 13, these anomalies are shown by the bold-faced symbols in the AEP which are observed when the $\Sigma \mathbf{D}(j, y)$ 's in Fig. 6 show their phase shift toward early hours. Unlike the harmonious long-term variations among $\Sigma \mathbf{D}(j, y)$ 's at the six stations in Fig. 6, the anomalies in Fig. 13 are not harmonious to each other. These incoherent movements, however, provide rather helpful leads to detect $\mathbf{D}(j, y)_{\mathrm{TSO}}$ in $\mathbf{D}(j, y)$, as they resemble those of the simulated $\mathbf{D}(j, y)_{\mathrm{TD}}$ with $\beta_{0}<1$ in Fig. 11 in their form that: 


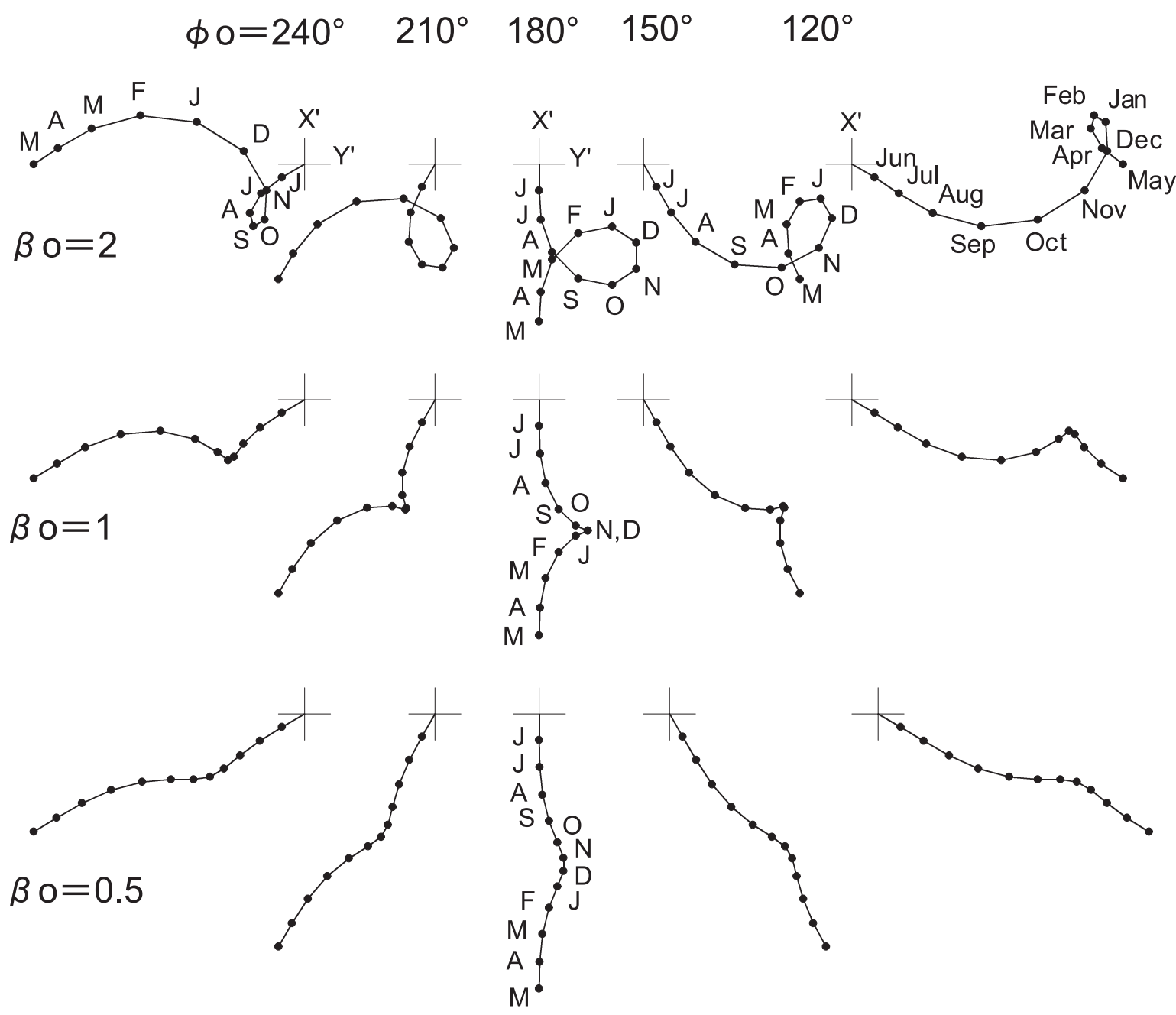

Fig. 11. Simulation of $\Sigma \mathbf{D}(j, y)_{\text {TD }}$ produced by $\mathbf{D}(j, y)_{\mathrm{TD}}$ in Eq. (9) for various combinations of $\beta_{0}$ and $\phi_{0}$ in Eq. (10).

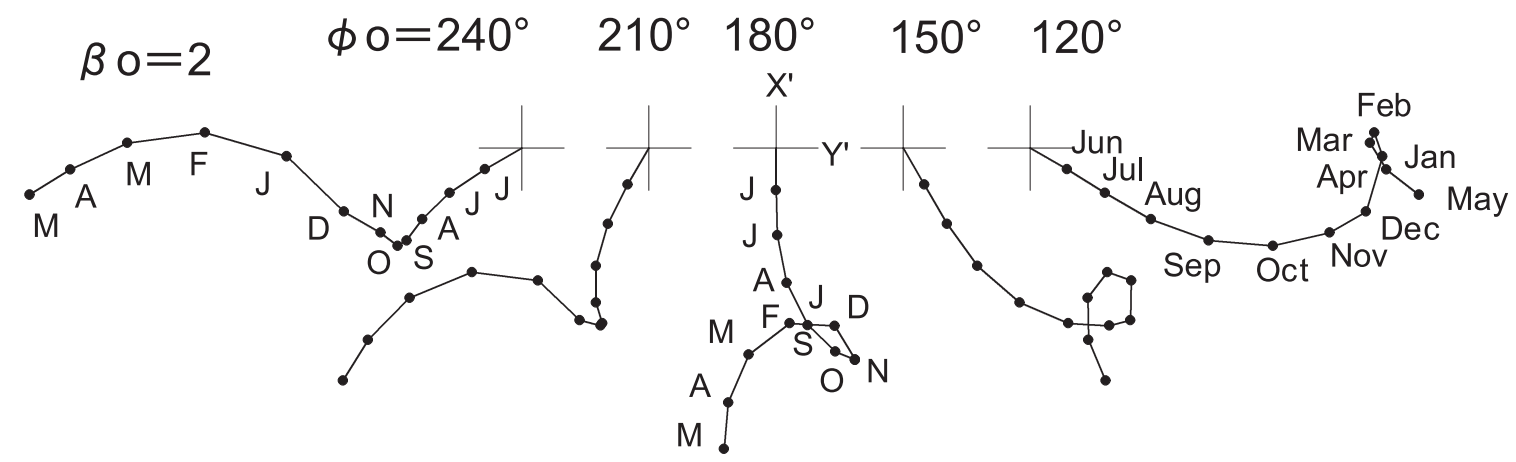

Fig. 12. Simulation of $\Sigma \mathbf{D}(j, y)_{\mathrm{TD}}$ produced by the modulated $\mathbf{F}(y)_{\mathrm{TSO}}$ in Eq. (11) for $\beta_{0}=2$ and several $\phi_{0}$ 's.

(1) Their phase $\phi(j, y)$ in the second quadrant $\left(90^{\circ}-180^{\circ}\right)$ of the harmonics coordinates in Fig. 13 shows the morning-type variation, while in the third quadrant $\left(180^{\circ}-270^{\circ}\right)$ it shows the evening-type.

(2) The morning- and evening-type variations are enhanced with the advancement and the retardation, respectively, of $\phi(j, y)$.

(3) At the boundary between the morning- and eveningtype regions, the variation of $\mathbf{D}(j, y)$ is small, as is observed at Rome in Fig. 13, indicating the transition between the two types.
These characteristics are most clearly observed in the variations from late 1993 to early 1994 in Fig. 13 and can safely identify them as being due to the phase anomaly. Among these variations, the one at Beijing stands out. As has been pointed out previously, this kind of variation at Beijing can be observed almost every year in the P-state (1989-2000), with its maximum in 1993-1994 (cf. Fig. 6). Nevertheless, the phase anomaly was discovered first in the solar activity minimum period (1995-1996) when the phase anomaly at Beijing shows rather a negligibly small value. This is due to the following reason. The enhance- 


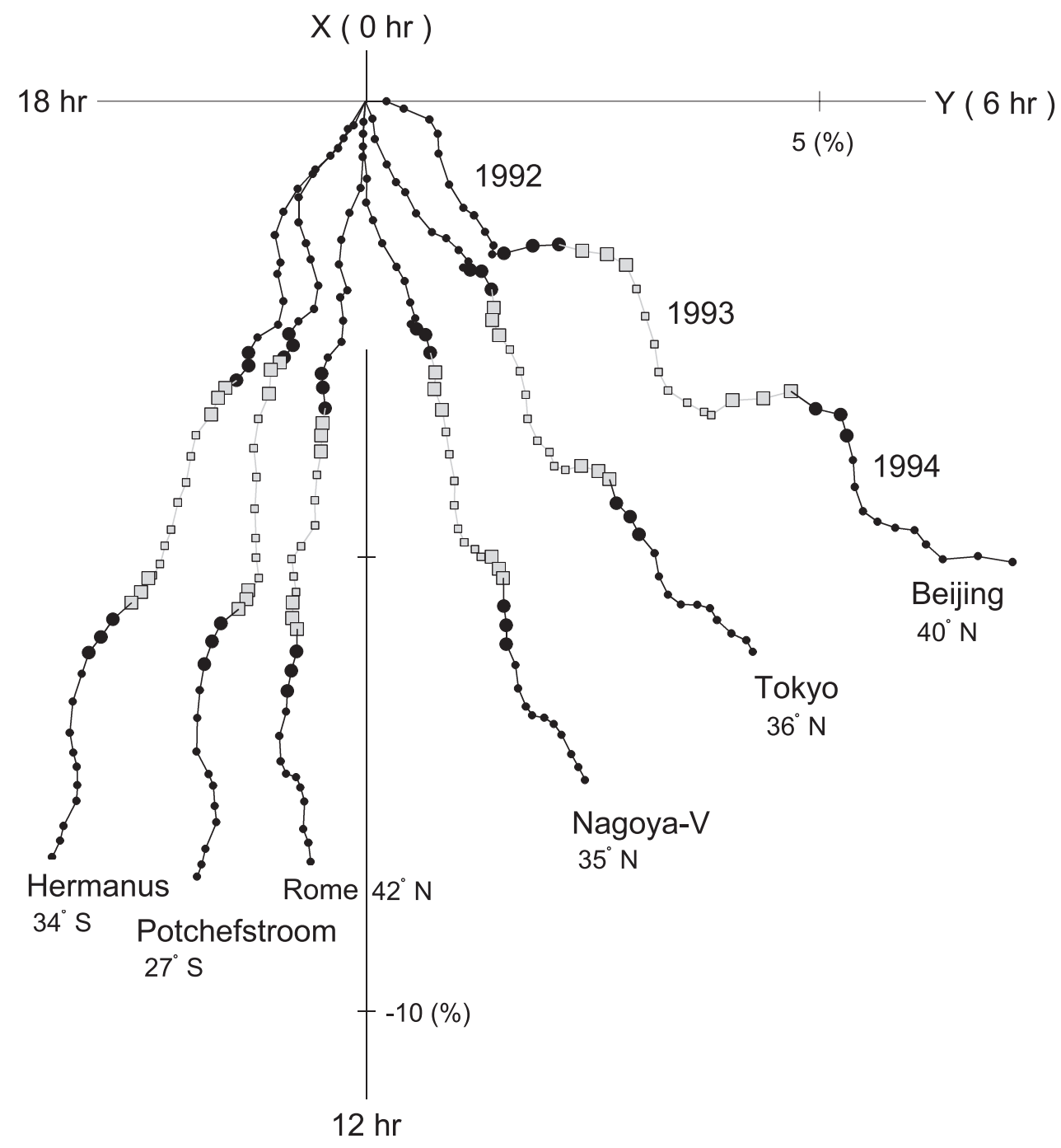

Fig. 13. Two consecutive phase anomalies of $\Sigma \mathbf{D}(j, y)$ in the AEP's shown by the bold-faced symbols in the active period of solar cycle (1992-1994) in the P-state.

ment of the phase anomaly is proportional to the ratio $\beta\left[=\mathrm{D}(j, y)_{\mathrm{TSO}} / \mathrm{D}(j, y)_{\mathrm{DC}}\right]$, which is variable depending on the cut-off rigidity at each station. In the solar activity maximum periods, the enhancement of the phase anomaly in the low latitude region (Beijing) can be observed, but the one in the high latitude region is difficult to be recognized, as $\mathbf{D}(j, y)_{\mathrm{DC}}$ in the region is very much larger than that at low latitudes (cf. Fig. 6). On the contrary, in the solar activity minimum period, although $\mathbf{D}(j, y)_{\text {TSO }}$ becomes smaller with the decrease of the solar activity (Nagashima et al., 2004, 2005), the corresponding $\mathbf{D}(j, y)_{\mathrm{DC}}$ also decreases much more severely and produces large values of $\beta$, especially in high latitudes (cf. Fig. 6). This causes clear enhancement of the phase anomaly in almost all of the latitude regions in the solar activity minimum period (1995-1996), (cf. Fig. 7).

For the transition from the P-state to the N-state, $\mathbf{F}(y)_{\mathrm{T}}$ reduces its magnitude and another anisotropy called $\mathbf{F}(y)_{\mathrm{H}}$ would increase in magnitude. The sharply concentrated $\mathbf{F}_{\mathrm{H}}$, produced by the cosmic-ray acceleration on the helionose boundary $\left(\alpha_{\mathrm{H}}=18 \mathrm{~h}\right)$, has always been observed by the neutron monitors being accompanied with $\mathbf{F}_{\mathrm{T}}$. Therefore,
$\mathbf{F}(y)_{\text {HSO }}$ could be found in the solar diurnal variation in the summer season ( $\sim$ June solstice) in the N-state when it is much enhanced by the acceleration of cosmic rays (cf. Nagashima et al., 2005; Nagashima and Fujii, 2006). If the seasonal variation of $\mathbf{F}(y)_{\mathrm{H}}$ is similar to that of $\mathbf{F}(y)_{\mathrm{T}}$, $\mathbf{F}(y)_{\text {HSO }}$ shows the maximum and minimum at the June and December solstices, respectively, and its yearly average $\mathbf{F}_{\mathrm{HSO}}$ is in the same direction as that $(0 \mathrm{~h} \mathrm{LT})$ of $\mathbf{F}_{\mathrm{TSO}}$. $\mathbf{F}(y)_{\mathrm{HSO}}$ produces $\mathbf{D}(j, y)_{\mathrm{HSO}}$ similar to $\mathbf{D}(j, y)_{\mathrm{TSO}}$ in Eq. (7), as

$$
\begin{aligned}
& \mathrm{D}(j, y)_{\mathrm{HSO}}=\zeta(j, y)_{\mathrm{HSO}} \mathrm{F}(y)_{\mathrm{HSO}} \\
& \phi(j, y)_{\mathrm{HSO}}=\Phi(y)_{\mathrm{HSO}}+\Delta \phi_{\mathrm{g}}(j, y)_{\mathrm{HSO}} .
\end{aligned}
$$

$\mathbf{D}(j, y)_{\mathrm{HSO}}$ produces the $\mathrm{H}$-modulated variation $\mathbf{D}(j, y)_{\mathrm{HD}}$, as

$$
\mathbf{D}(j, y)_{\mathrm{HD}}=\mathbf{D}(j, y)_{\mathrm{HSO}}+\mathbf{D}(j, y)_{\mathrm{DC}}
$$

The seasonal variation of $\mathbf{D}(j, y)_{\mathrm{HSO}}$ in Eq. (12) describes the same curve as that of $\mathbf{D}(j, y)_{\text {TSO }}$ in Fig. 10, if the period of the month $M$ in the figure is replaced with $M+6$, as shown on the right at the top of Fig. 14. According to this 

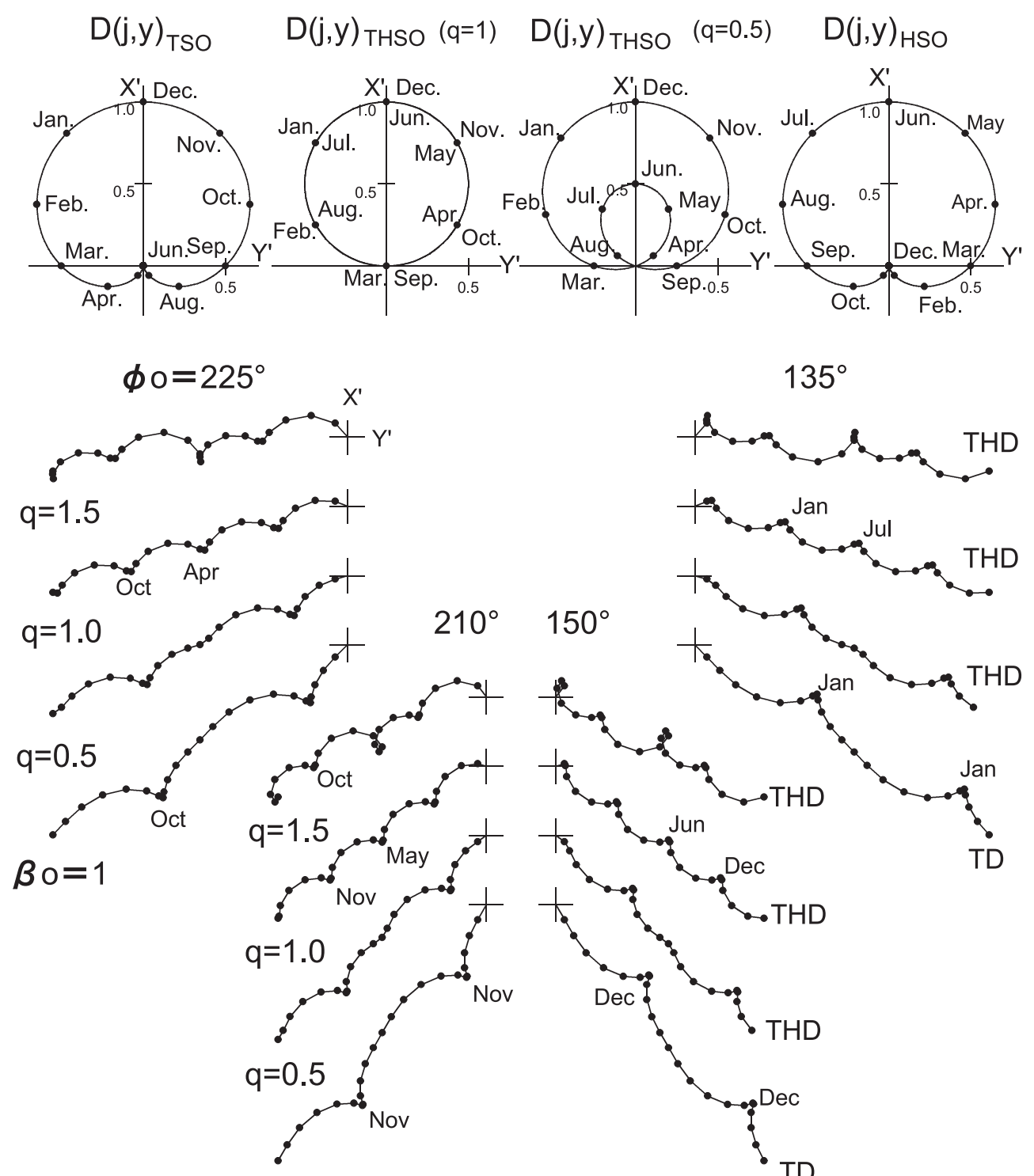

$135^{\circ}$

$\beta 0=1$

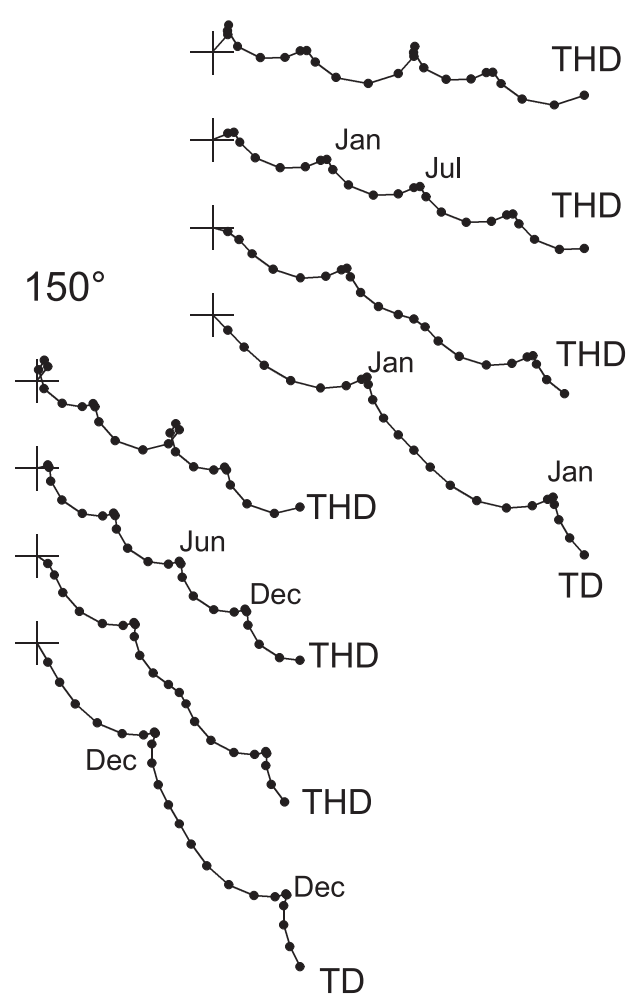

Fig. 14. The seasonal variations $\mathbf{D}(j, y)_{\text {TSO }}$ and $\mathbf{D}(j, y)_{\text {HSO }}$ with their maxima $\mathbf{D}(j, 0)_{\text {TSO }}=\mathbf{D}(j, 0.5)_{\text {HSO }}=1$ respectively at the December and June solstices are shown on the left and right side at the top, and their interaction $\mathbf{D}(j, y)_{\mathrm{THSO}}$ with $q\left(=\mathrm{D}(j, 0.5)_{\mathrm{HSO}} / \mathrm{D}(j, 1)_{\mathrm{TSO}}\right)=1$ and 0.5 are in the middle part. On the lower part, an example of $\Sigma \mathbf{D}(j, y)_{\mathrm{TD}}$ in Eq. (9) with $\beta_{0}=1$ in Eq. (10) is at the bottom, and $\mathbf{D}(j, y)_{\mathrm{THD}}$ 's in Eq. (14) produced by the interaction of $\mathbf{D}(j, y)_{\mathrm{HSO}}$ with $\mathbf{D}(j, y)_{\mathrm{TD}}$ for the cases of $q=0.5,1$ and 1.5 are shown in the middle. All the $\Sigma \mathbf{D}(j, y)_{\mathrm{TD}}$ and $\Sigma \mathbf{D}(j, y)_{\mathrm{THD}}$ start from the June solstice. Alphabetical marks express respectively $\Sigma \mathbf{D}(j, y)_{\mathrm{TD}}$ and $\Sigma \mathbf{D}(j, y)_{\mathrm{THD}}$.

replacement, the simulation of $\Sigma \mathbf{D}(j, y)_{\mathrm{HD}}$ in Fig. 12 also shows the same variation as that of $\Sigma \mathbf{D}(j, y)_{\mathrm{TD}}$ in Fig. 11 for any combination of $\phi_{0}$ and $\beta_{0}$ defined by the replacement of $\mathbf{D}(j, y)_{\text {TSO }}$ in Eq. (10) with $\mathbf{D}(j, y)_{\text {HSO }}$. If, however, $\mathbf{F}(y)_{\mathrm{HSO}}$ and $\mathbf{F}(y)_{\mathrm{TSO}}$ coexist in a year, $\mathbf{D}(j, y)_{\mathrm{HSO}}$ and $\mathbf{D}(j, y)_{\text {TSO }}$ interact with each other, the examples of which are shown in the middle at the top of Fig. 14 in the cases that $q\left(=\mathrm{D}(j, 0.5)_{\mathrm{HSO}} / \mathrm{D}(j, 0)_{\mathrm{TSO}}\right)$ is 1 and 0.5 . These two vectors produce the TH-modulated variation $\mathbf{D}(j, y)_{\mathrm{THD}}$ as

$$
\mathbf{D}(j, y)_{\mathrm{THD}}=\mathbf{D}(j, y)_{\mathrm{TSO}}+\mathbf{D}(j, y)_{\mathrm{HSO}}+\mathbf{D}(j, y)_{\mathrm{DC}} .
$$

Some examples of $\Sigma \mathbf{D}(j, y)_{\mathrm{THD}}$ are shown on the lower part of Fig. 14 together with $\Sigma \mathbf{D}(j, y)_{\mathrm{TD}}$ for the comparison between them. The mutual interaction between $\mathbf{D}(j, y)_{\text {TSO }}$

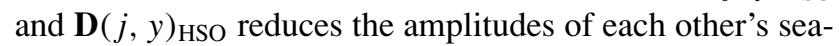

sonal variation, but it escalates the phase advancement of the morning-type variation and the phase retardation of the evening-type variation, respectively, on a yearly basis. This is due to the following fact that the two sidereal anisotropies $\mathbf{F}(y)_{\mathrm{T}}$ and $\mathbf{F}(y)_{\mathrm{H}}$ from the opposite right ascensions of $6 \mathrm{~h}$ and $18 \mathrm{~h}$ produce the yearly averaged $\mathbf{F}_{\mathrm{TSO}}$ and $\mathbf{F}_{\mathrm{HSO}}$, respectively, from the common $0 \mathrm{~h} \mathrm{LT}$ in the solar geographic coordinate, as has been stated in Introduction.

The existence of $\mathbf{D}(j, y)_{\mathrm{THD}}$ was observed in the solar activity minimum period (1985-1987) in the N-state. Some examples of the variations are shown by the bold-faced symbols in $\Sigma \mathbf{D}(j, y)$ 's drawn separately in Fig. 15(A) for the nine northern and southern high latitude stations and in Fig. 15(B) for the three low latitude stations. $\mathbf{D}(j, y)_{\mathrm{HSO}}$ and $\mathbf{D}(j, y)_{\text {TSO }}$ interact with each other and their AEP's are from April to September and from October to March, 


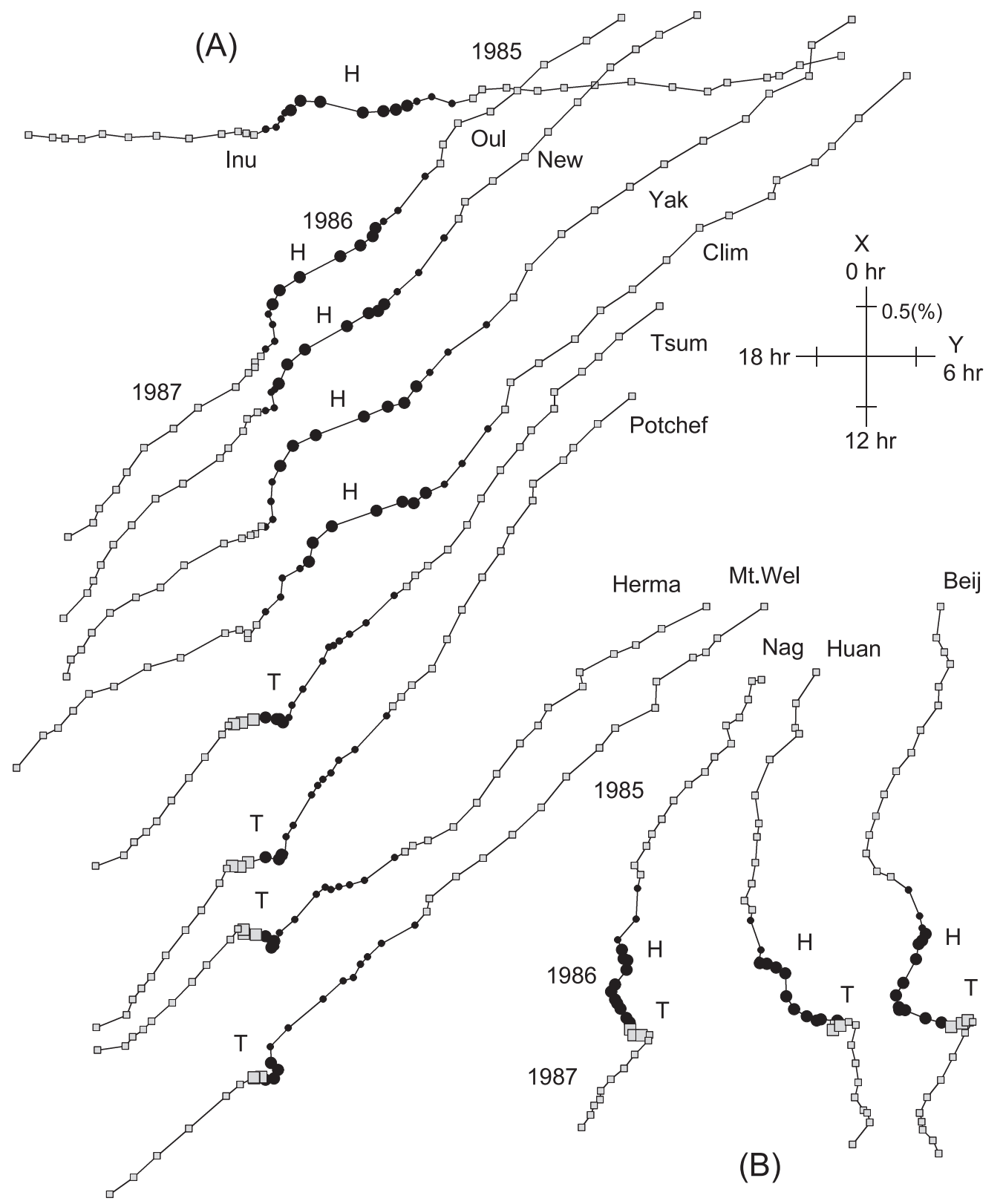

Fig. 15. $\Sigma \mathbf{D}(j, y)$ 's in the N-state (1985-1987) at the nine high latitudes stations at the top (A) and those in the three low latitude stations at the bottom (B). The influences of $\mathbf{D}(j, y)_{\text {TSO }}$ and $\mathbf{D}(j, y)_{\text {HSO }}$ due to $\mathbf{F}(y)_{\text {TSO }}$ and $\mathbf{F}(y)_{\text {HSO }}$ are shown by the bold-faced symbols with the labels $\mathrm{T}$ and $\mathrm{H}$ in the respective AEP's, (cf. text). Inu: Inuvik $\left(68^{\circ} \mathrm{N}\right)$, Oul: Oulu $\left(65^{\circ} \mathrm{N}\right)$, New: Newark $\left(40^{\circ} \mathrm{N}\right)$, Yak: Yakutsk $\left(62^{\circ} \mathrm{N}\right)$, Clim: Climax $\left(39^{\circ} \mathrm{N}\right)$, Tsum: Tsumeb $\left(19^{\circ} \mathrm{S}\right)$, Potchef: Potchefstroom $\left(27^{\circ} \mathrm{S}\right)$, Herma: Hermanus $\left(34^{\circ} \mathrm{S}\right)$, Mt. Wel: Mt. Wellington $\left(43^{\circ} \mathrm{S}\right)$, Nag: Nagoya $\left(35^{\circ} \mathrm{N}\right)$, Huan: Huancayo $\left(12^{\circ} \mathrm{S}\right)$, Beij: Beijing $\left(40^{\circ} \mathrm{N}\right)$.

respectively. Owing to the mutually reversed N-S asymmetric latitude distributions of $\mathbf{F}(y)_{\mathrm{HSO}}$ and $\mathbf{F}(y)_{\text {TSO }}$ (cf. Nagashima et al., 1998; Nagashima and Fujii, 2006), $\mathbf{D}(j, y)_{\text {HSO }}$ dominates in the northern hemisphere, while $\mathbf{D}(j, y)_{\text {Tso }}$ dominates in the southern hemisphere. Therefore, the clear appearance of the phase anomalies of the two types can not always be expected at just any station, except those in low latitudes, such as those stations Beijing, Tokyo, and Nagoya shown in Fig. 15(B). At these three stations, $\mathbf{D}(j, y)_{\text {HSO }}$ with the mark $\mathrm{H}$ and $\mathbf{D}(j, y)_{\text {TSO }}$ with the mark $\mathrm{T}$ show the morning-type variations with their respective maximum peaks near the June and December solstices; they also show their mutual interaction similar to that of the simulation in Fig. 14. In high latitudes, on the other hand, the mutual interaction is reduced, and $\mathbf{D}(j, y)_{\mathrm{HSO}}$ and $\mathbf{D}(j, y)_{\text {TSO }}$ of the evening-type are enhanced in the northern and southern hemispheres, respectively, as shown by the respective marks $\mathrm{H}$ and $\mathrm{T}$ in Fig. 15(A). Among them, the most explicit variations with the mark $\mathrm{T}$ are observed at the southern stations Tsumeb $\left(19^{\circ} \mathrm{S}\right)$, Potchefstroom $\left(27^{\circ} \mathrm{S}\right)$, and Hermanus $\left(34^{\circ} \mathrm{S}\right)$. Unlike those in the P-state, the variations are limited only in the narrow southern latitude region where $R(j)$ of $\mathbf{D}(j, y)$ in the P-state (1995-1996) shows the maximum (cf. Fig. 8). This is due to the fact that $\mathbf{D}(j, y)_{\text {TSO }}$ in the $\mathrm{N}$-state becomes smaller than when it is in the P-state (cf. Nagashima et al., 2004). The transition from the looptype in the P-state to the evening-type in the $\mathrm{N}$-state is due to the shift of $\phi(j)_{\mathrm{DC}}$ from the noon-side toward the af- 
ternoon side (cf. Figs. 6 and 14). Unlike the $\mathbf{D}(j, y)_{\mathrm{TSO}}$ in 1995-1996, the present one starts earlier than January, indicating less deformation from the axis-symmetric distribution of $\mathbf{F}(y)_{\text {TSO }}$. On the other hand, $\mathbf{F}(y)_{\text {HSO }}$ produces the evening-type $\mathbf{D}(j, y)_{\text {HSO }}$ with the maximum at the June solstice in northern high latitudes, shown by the bold-faced symbols with the mark $\mathrm{H}$ in Fig. 15(A). The magnitude $R(j)$ of the total sum of $\mathbf{D}(j, y)$ 's in the respective duration periods of $\mathbf{D}(j, y)_{\mathrm{HSO}}$ is at a maximum in the northern hemisphere and rapidly diminishes in the southern hemisphere. As above, any station in the high latitudes observes only a single enhanced anomaly, shown by the bold-faced symbols with the mark $\mathrm{H}$ or $\mathrm{T}$. However, some northern stations seem to observe a sign of the beginning of another anomaly right after the end of the AEP of the previous anomaly due to $\mathbf{D}(j, y)_{\mathrm{HSO}}$. Generally, the eveningtype phase anomaly of $\mathbf{D}(j, y)_{\mathrm{HSO}}$ on the harmonic diagram describes a smooth trajectory almost parallel to that of $\mathbf{D}(j, y)_{\mathrm{DC}}$ after the end of the September (cf. Fig. 11). The observed trajectory, however, changes suddenly after this period: the trajectory shifts downwards for the first several months, stagnates there for some months with small magnitudes, and then shifts upwards, recovering its magnitude and direction. It should be noted that the stagnation period almost coincides with that of the $\mathbf{D}(j, y)_{\text {TSO }}$ observed at the southern stations Tsumeb, Potchefstroom, and Hermanus (cf. Fig. 15), and furthermore, that these successive downwards, stagnant, and upwards movements of the trajectory almost coincide with the simulation produced by the interaction between $\mathbf{D}(j, y)_{\text {HSO }}$ and $\mathbf{D}(j, y)_{\text {TSO }}$ shown in Fig. 14. This indicates that the part of this period in Fig. 15 would be produced by $\mathbf{D}(j, y)_{\text {Tso. }}$

The above analysis reveals that the two anisotropies $\mathbf{F}_{\mathrm{T}}$ and $\mathbf{F}_{\mathrm{H}}$ would influence the solar anisotropy $\left(\mathbf{F}_{\mathrm{DC}}\right)$ and would produce the previous D-anomaly in Section 1. We now attempt to explain the D-anomaly by using $\mathbf{F}_{\mathrm{T}}$ as a representative for the two anisotropies.

As mentioned earlier, the annual variation $\mathbf{F}(y)$ TSO produces the yearly averaged $\mathbf{F}_{\mathrm{TSO}}$ from the direction of $0 \mathrm{~h} \mathrm{LT}$. The resultant $\left(\mathbf{F}_{\mathrm{TD}}\right)$ of $\mathbf{F}_{\mathrm{TSO}}$ and $\mathbf{F}_{\mathrm{DC}}$ produces the following T-modulated solar diurnal variations.

$$
\mathbf{D}(j)_{\mathrm{TD}}=\mathbf{D}(j)_{\mathrm{TSO}}+\mathbf{D}(j)_{\mathrm{DC}},
$$

which is the yearly average of Eq. (9). The schematic representations of the relative configurations of $\mathbf{D}(j)_{\mathrm{TD}}, \mathbf{D}(j)_{\mathrm{DC}}$ and $\mathbf{D}(j)_{\text {TSO }}$ are shown in Fig. 16 for the three different $\Delta \phi(j)_{\mathrm{TD}}$ 's (A, B, and C) defined by

$$
\Delta \phi(j)_{\mathrm{TD}}=\phi(j)_{\mathrm{TSO}}-\phi(j)_{\mathrm{DC}} .
$$

Owing to the introduction of $\mathbf{D}(j)_{\mathrm{TSO}}$, the normalized vector must be changed from $\overline{\mathbf{D}}(j)_{\mathrm{DC}}$ to $\overline{\mathbf{D}}(j)_{\mathrm{TD}}$ defined by (cf. Eqs. (4) and (5))

$$
\begin{aligned}
& \overline{\mathrm{D}}(j)_{\mathrm{TD}}=\mathrm{D}(j)_{\mathrm{TD}} / \mathrm{D}(\mathrm{INU})_{\mathrm{TD}} \fallingdotseq \mathrm{D}(j)_{\mathrm{TD}} / \mathrm{D}(\mathrm{INU})_{\mathrm{DC}} \\
& \bar{\phi}(j)_{\mathrm{TD}}=\phi(j)_{\mathrm{TD}}-\phi(\mathrm{INU})_{\mathrm{TD}} \fallingdotseq \phi(j)_{\mathrm{TD}}-\phi(\mathrm{INU})_{\mathrm{DC}}
\end{aligned}
$$

in which the approximation is permissible as $\mathbf{D}(\mathrm{INU})_{\text {TSO }}$ is negligibly small owing to the north-south asymmetry of the

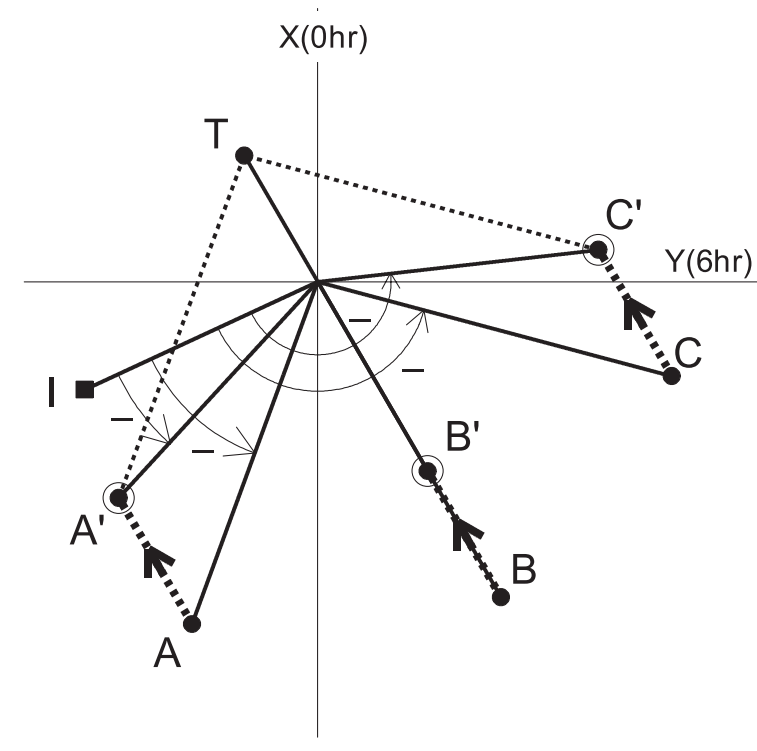

Fig. 16. The transition from $\mathbf{D}(j)_{\mathrm{DC}}$ to $\mathbf{D}(j)_{\mathrm{TD}}$ by the influence of

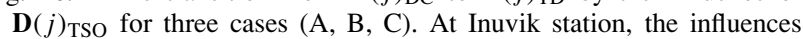
of $\mathbf{D}(j)_{\text {TSO }}$ is neglected as it is very small. The arrow expresses the transition. A, B, and C: $\mathbf{D}(j)_{\mathrm{DC}}, \mathrm{A}^{\prime}, \mathrm{B}^{\prime}$, and $\mathrm{C}^{\prime}: \mathbf{D}(j)_{\mathrm{TD}}, \mathrm{T}: \mathbf{D}(j)_{\mathrm{TSO}}$, and I: $\mathbf{D}(\mathrm{INUVIK})_{\mathrm{TD}} \fallingdotseq \mathbf{D}(\mathrm{INUVIK})_{\mathrm{DC}}$.

T-anisotropy (cf. Fig. 8). Because of this negligible influence of $\mathbf{D}(\mathrm{INU})_{\mathrm{TSO}}$ on $\mathbf{D}(\mathrm{INU})_{\mathrm{DC}}$, the relative position between $\mathbf{D}(j)_{\mathrm{TD}}$ and $\mathbf{D}(j)_{\mathrm{DC}}$ on the $\overline{\mathrm{D}}-\bar{\phi}$ diagram can be easily estimated in Fig. 16. In the case of A when $\Delta \phi(j)_{\mathrm{TD}}<\pi$, $\bar{\phi}(j)_{\mathrm{TD}}$ shifts toward the evening side from $\bar{\phi}(j)_{\mathrm{DC}}$. This relation produces the leftward deviation of $\overline{\mathbf{D}}(j)$ from the net area, as can be seen at Hermanus in Fig. 5. Almost all the $\overline{\mathbf{D}}$ (Hermanus)s in the left outside of the net indicates that the influence of $\mathbf{F}_{\mathrm{TSO}}$ exists during almost all of the years, as expected. On the other hand, in the case of $\mathrm{C}$ when $\Delta \phi(j)_{\mathrm{TD}}>\pi, \bar{\phi}(j)_{\mathrm{TD}}$ shifts toward the morning side from $\bar{\phi}(j)_{\mathrm{DC}}$. This relation produces the rightward deviation of $\overline{\mathrm{D}}(j)$ from the net area, as can be seen at Nagoya in Fig. 5, but not all the time because the above condition is realized mainly in the solar activity minimum period in the P-state when $\phi(j)_{\mathrm{DC}}$ shifts toward the morning side, showing a good agreement with the observation. Finally, in the case of $\mathrm{B}$ when $\Delta \phi(j)_{\mathrm{TD}} \approx \pi$, no phase shift occurs, but $\mathbf{D}(j)_{\mathrm{DC}}$ changes its magnitude to a small $\mathbf{D}(j)_{\mathrm{TD}}$. This phenomenon can also be seen in the distribution of $\overline{\mathbf{D}}$ (Hermanus)'s in Fig. 5. Although almost all the vectors in Fig. 5 are in the forbidden region, with the exception of vectors in 1996 and 1997, which are out of the group and show very small amplitudes. The amplitude in 1996 is, in particular, the smallest among those at all the other stations. These facts suggest that case B is realized by the shift of $\phi(j)_{\mathrm{DC}}$ from the A-state to the B-state in Fig. 16 in the solar activity minimum period in the Pstate. Note that, in the other cases (A and $\mathrm{C}$ ), the magnitude $\mathrm{D}(j)_{\mathrm{TD}}$ depends not only on $\Delta \phi(j)_{\mathrm{TD}}$ but also on the ratio $\mathrm{D}(j)_{\mathrm{TSO}} / \mathrm{D}(j)_{\mathrm{DC}}$. It is noted also that if $\mathbf{D}(j)_{\mathrm{HSO}}$ due to $\mathbf{F}_{\mathrm{H}}$ is superposed on $\mathbf{D}(j)_{\mathrm{TsO}}$, Eq. (15) is changed to the yearly average of Eq. (14), and $\mathbf{D}(j)_{\text {TSO }}$ with the mark T in Fig. 16 is replaced with $\left(\mathbf{D}(j)_{\mathrm{TSO}}+\mathbf{D}(j)_{\mathrm{HSO}}\right)$. If we tentatively assume for simplicity that the deflection angles 


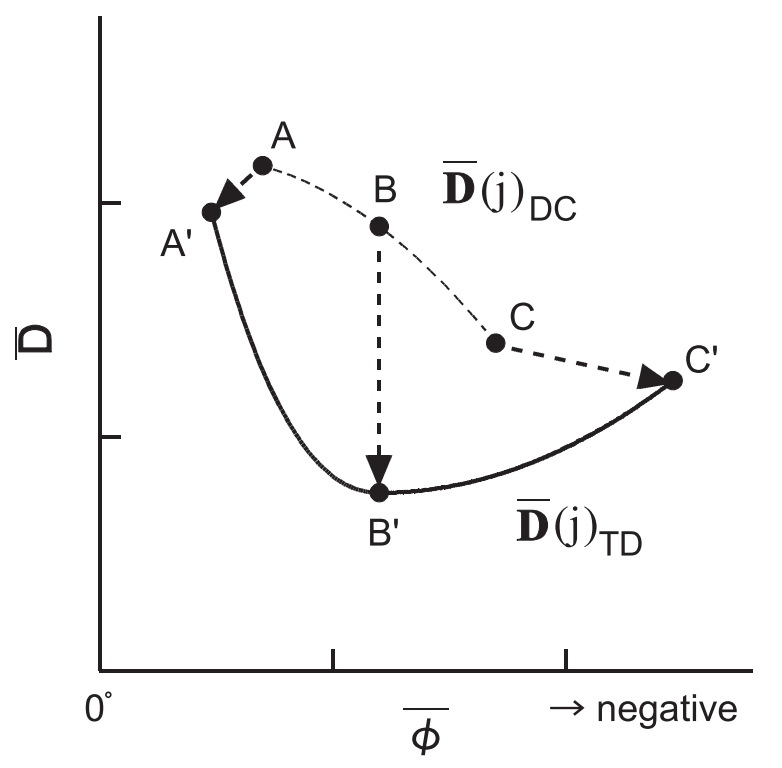

Fig. 17. The schematic distribution of $\overline{\mathbf{D}}(j)_{\mathrm{TD}}$ 's and $\overline{\mathbf{D}}(j)_{\mathrm{DC}}$ 's on $\overline{\mathrm{D}}-\bar{\phi}$-diagram drawn respectively with the solid and dotted lines. The arrow expresses the transition from $\overline{\mathbf{D}}(j)_{\mathrm{DC}}$ to $\overline{\mathbf{D}}(j)_{\text {TD }}$ according to the rule shown in Fig. 16.

$\Delta \phi_{\mathrm{g}}(j)_{\mathrm{TSO}}$ and $\Delta \phi_{\mathrm{g}}(j)_{\mathrm{HSO}}$ are the same as each other, the interaction between $\mathbf{F}_{\mathrm{T}}$ and $\mathbf{F}_{\mathrm{H}}$ increases only the amplitude $\mathrm{T}$ in Fig. 16 and escalates the three types (A, B, and C) of $\mathrm{D}$-anomaly, as has been already shown by the seasonal variation in Fig. 14.

The configuration between $\overline{\mathbf{D}}(j)_{\mathrm{DC}}$ and $\overline{\mathbf{D}}(j)_{\mathrm{TD}}$ in Fig. 16 explains also the D-anomaly in Figs. 3 and 4 . The $\overline{\mathrm{D}} \cdot \bar{\phi}$ diagrams of the schematic distributions of $\overline{\mathbf{D}}(j)_{\mathrm{TD}}$ and $\overline{\mathbf{D}}(j)_{\text {DC }}$ are drawn with solid and dotted lines, respectively, in Fig. 17. The arrows with $\mathrm{A}, \mathrm{B}$, or $\mathrm{C}$ express the transition from $\overline{\mathbf{D}}(j)_{\mathrm{DC}}$ to $\overline{\mathbf{D}}(j)_{\mathrm{TD}}$ according to the rule shown in Fig. 16. In this way, the D-anomaly is solved by the introduction of $\overline{\mathbf{D}}(j)_{\mathrm{TSO}}$.

\section{Discussion}

It is well known that the solar diurnal variation $\mathbf{D}(j)$ of cosmic rays shows a two-solar-cycle periodicity in that its phase $\phi(j)$ shifts toward the morning side in the positive (P-) polarity state of the solar polar magnetic field, while in the negative $(\mathrm{N}-)$ state it shifts toward the evening side (cf. Figs. 1 and 2). As stated in Introduction, such a polarity dependence of $\phi(j)$ was thought to be fully explained by the effect of the polarity-dependent heliolatitudinal drift motion of cosmic rays pointed out by Jokipii and Kopriva (1979). In the recent P-state (1990 2000), the phase shift toward the morning side became very much larger than that in the preceding P-state (1970 1980), and in the solar activity minimum period (1996), the phase showed a strange behavior that can not be explained by the diffusion-convection theory. This phenomenon was first found in the diurnal variations $\mathbf{D}(j)$ 's observed with the vertical $(\mathrm{V})$ and four directional muon telescopes (N, E, S, W) set at zenith angle $30^{\circ}$ and azimuthal angles $0^{\circ}, 90^{\circ}, 180^{\circ}$ and $270^{\circ}$ at Nagoya $\left(35^{\circ} \mathrm{N}, 137^{\circ} \mathrm{E}\right)$, (cf. Fujii et al., 1996, 2000). These variations are plotted by the points on the diurnal harmonic coordinate in Fig. 18(A). In order to emphasize the relative configuration ( $\mathrm{RC})$ of $\mathbf{D}(j)$ 's, each vector is connected with the neighboring points by the solid lines, like a constellation in the sky, except for the V-vector, which is connected with the origin $\mathrm{O}$. The direction $\Phi$ of the anisotropy responsible for these $\mathbf{D}(j)$ 's in the figure is estimated from the observation of $\phi$ (INU) in 1996 using Eq. (4). Corresponding to this $\mathrm{RC}$, the simulations of the configuration $\left(\mathrm{RC}_{\mathrm{DC}}\right)$ in Fig. 18(C) are those due to $\mathbf{F}_{\mathrm{DC}}$ with the spectra for several combinations of $\gamma$ and $p_{\mathrm{u}}$ from the tentative direction of $\Phi_{\mathrm{DC}}=12 \mathrm{~h} \mathrm{LT}$ (cf. Fujimoto et al., 1984). The observed RC of Fig. 18(A) is quite contradictory to those of the simulations, because when $\mathrm{RC}$ fits with $\mathrm{RC}_{\mathrm{DC}}$ in the case b, $\left|\Delta \phi_{\mathrm{g}}(\mathrm{V})\right|$ is too large to coincide with $\left|\Delta \phi_{\mathrm{g}}(\mathrm{V})_{\mathrm{DC}}\right|$. Conversely, when $\left|\Delta \phi_{\mathrm{g}}(\mathrm{V})\right|$ coincides with $\left|\Delta \phi_{\mathrm{g}}(\mathrm{V})_{\mathrm{DC}}\right|$ in the case e or $\mathrm{f}, \mathrm{RC}$ does not fit with $\mathrm{RC}_{\mathrm{DC}}$. On the contrary, another RC of $\mathbf{D}(j)$ 's in the solar activity minimum period (1986) in the N-state shown in Fig. 18(B) fits well with the simulation in the case of $\mathrm{d}$. This agreement provides evidence of the reliability of the observation, and proves that the disagreement between $\mathrm{RC}$ and $\mathrm{RC}_{\mathrm{DC}}$ in 1996 is not due to observational error. Its universality was also confirmed soon afterwards by the finding of the abnormally early appearance of $\phi(j)$ toward the morning side at limited neutron monitor stations with comparatively high cut-off rigidities $\left(p_{\mathrm{c}}\right)$ at low latitudes.

One solution for this difficulty was to assume some modification of the parameters in the diffusion-convection equation. One of the parameters which can change the direction $\Phi$ of the anisotropy is contained in the scattering mean free path $L(p, \Lambda)$ of cosmic rays (cf. Garcia-Munoz et al., 1975; McDonald et al., 1977), as

$$
L(p, \Lambda) \propto 1+a_{\mathrm{L}} \sin \Lambda,
$$

where $\Lambda$ is the heliolatitude and $a_{\mathrm{L}}$ is a constant parameter. The shift of $\Phi$ toward the morning side in the P-state was thought to be produced by the increase of $a_{\mathrm{L}}$ with the decrease of solar activity in the P-state (cf. Munakata and Nagashima, 1986). If $a_{\mathrm{L}}$ is an increase function of $p$ such as

$$
a_{\mathrm{L}} \propto p^{\Gamma(>0)}
$$

the direction $\Phi$ of the anisotropy would be a function of $p$ and shift continuously toward the morning side with the increase of $p$-even in a definite period. Owing to this $\Phi(p)$ distribution, the anisotropy with a hard spectrum in high rigidities could produce $\mathbf{D}(j)$ 's of the clear rectangular type RC in the early morning side ( $\sim 6 \mathrm{~h} \mathrm{LT})$ despite the small $\left|\Delta \phi_{\mathrm{g}}(j)\right| \mathrm{s}$. Such an anisotropy with $\Phi(p)$ continuously changeable with $p$ is called the multi-directional anisotropy for simplicity. The solution for such a diffusionconvection equation has not been obtained. However, the multi-directional anisotropy can be expressed by the following 'dual anisotropy' which can estimate the solution without the aid of numerical equations.

If the diffusion-convection of cosmic rays in the HMS were not three-dimensional but limited only in the solar equatorial plane, such that the diffusion-in and the convection-out fluxes $\left(\mathbf{F}_{\mathrm{D}}\right.$ and $\left.\mathbf{F}_{\mathrm{C}}\right)$ of cosmic rays would arrive at the Earth from $21 \mathrm{~h} \mathrm{LT}$ along the magnetic field 
A) 1996

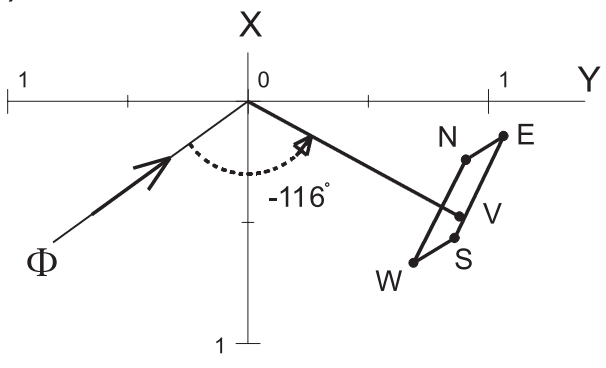

Simulation

C)

a) $r=0, P u=200$

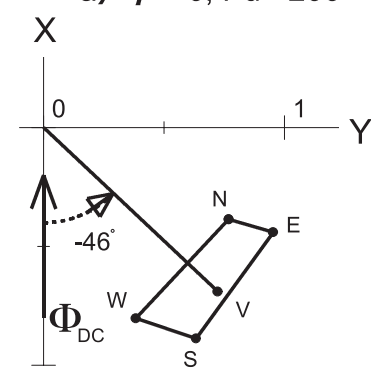

b) $r=0, P u=100$

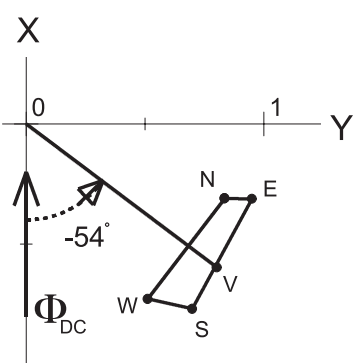

c) $r=0, \mathrm{Pu}=50$

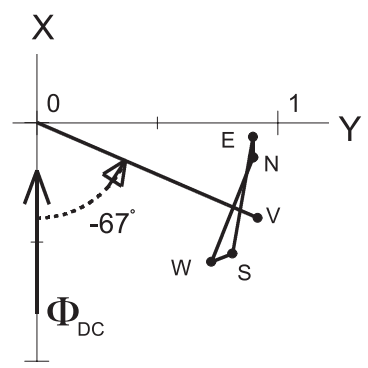

d) $r=0, P u=30$

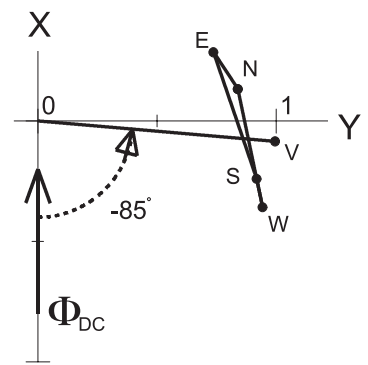

e) $r=-1, \mathrm{Pu}=30$

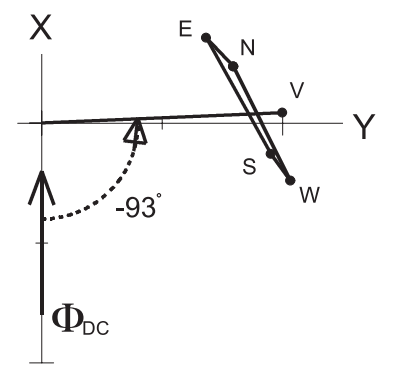

f) $r=-2, P u=30$

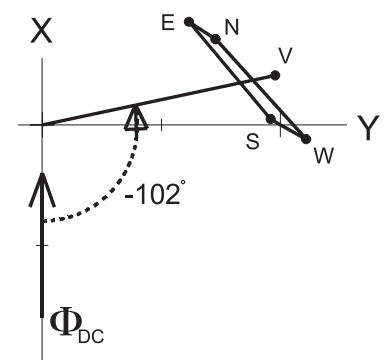

Fig. 18. The configuration of $\mathbf{D}(j)$ 's of five muon telescopes (V, N, E, S, W) at Nagoya observed in 1996 (A) and 1986 (B), and those derived theoretically from the solar anisotropy due to the diffusion-convection of cosmic rays with the spectrum with several combinations of $\gamma$ and $p_{\mathrm{u}}$ at the bottom $(\mathrm{C})$. The loop connecting the theoretical four vectors is clockwise in the order of $\mathrm{N}, \mathrm{E}, \mathrm{S}$, and $\mathrm{W}$ in the case of hard rigidity spectrum $(\gamma=0$ and $\left.p_{\mathrm{u}}>100 \mathrm{GV}\right)$, but in the case of the soft spectrum it is anticlockwise. In the intermediate rigidity region, the loop is in a twisted state. Note that, the magnitude of all the V-vectors in the figure are normalized to unity.

and $12 \mathrm{~h} \mathrm{LT}$ along the solar wind, respectively, the stationary (or quasi-stationary) state of the cosmic-ray density inside a circle around the Sun would have to be maintained by the compensation between $\mathbf{F}_{\mathrm{C}}$ and the radial component $\left(\mathbf{F}_{\mathrm{DR}}\right)$ of $\mathbf{F}_{\mathrm{D}}$. In this case, the resultant flux $\mathbf{F}_{\mathrm{DC}}\left(=\mathbf{F}_{\mathrm{D}}+\mathbf{F}_{\mathrm{C}}\right)$ would be always from the direction of $18 \mathrm{~h} \mathrm{LT}$ and could not produce the polarity dependence of $\Phi$. The dependence is produced by the three-dimensional diffusion-convection of cosmic rays in the HMS. In this case, a part of the diffusionin flux at high heliolatitudes in the P-state arrives at the equatorial region due to the heliolatitudinal drift motion of cosmic rays (Jokipii and Kopriva, 1979). This flux must be swept away from the sphere by an excess convectionout flux $\Delta \mathbf{F}_{\mathrm{C}}$ in the equatorial region in order to keep the cosmic-ray density in a stationary state in the sphere. This excess flux $\Delta \mathbf{F}_{\mathrm{C}}$ produces the phase shift from $18 \mathrm{~h}$ toward the morning side in cooperation with $\mathbf{F}_{\mathrm{DC}}$ in the above. The observed polarity dependence of $\Phi$ is thought to be realized by the increase of $\Delta \mathbf{F}_{\mathrm{C}}$ with the decrease of solar activity in the P-state owing to the structural change of the HMS (Munakata and Nagashima, 1986). If $\Delta \mathbf{F}_{\mathrm{C}}$ is rigiditydependent, such as

$$
\Delta \mathbf{F}_{\mathrm{C}}(p) \propto p^{\Gamma_{\mathrm{c}}(>0)},
$$

it produces the multi-directional anisotropy in any definite period. In other words, the multi-directional anisotropy can be expressed by a dual anisotropy $\mathbf{F}_{\mathrm{DC} 2}$ composed of the diffusion-in flux $\mathbf{F}_{\mathrm{D}}$ and the resultant convection-out flux $\mathbf{F}_{\mathrm{C}}+\Delta \mathbf{F}_{\mathrm{C}}$, having the soft and hard rigidity spectra $\left(f(p)_{\mathrm{D}}\right.$ and $\left.f(p)_{\mathrm{C}}\right)$, respectively, and arriving from $\Phi_{\mathrm{D}}(\sim 21 \mathrm{~h})$ and $\Phi_{\mathrm{C}}(\sim 12 \mathrm{~h})$, respectively. The dual anisotropy $\mathbf{F}_{\mathrm{DC} 2}$ could produce the required $\mathbf{D}(j)$ in Fig. 18(A) and those at neutron stations in low latitudes, but regrettably it also produced the similar but unwelcome variations at high-latitude 
neutron stations, whose phases were too early to coincide with those observed. This discrepancy in high latitudes could not be solved by any combination of the two spectra. Such a contradiction between the observation and the theory was the beginning of the discovery of the D-anomaly (cf. Figs. 3, 4 and 5), and the germ of the phase anomaly was also found in the same data as the phase shift of $\mathbf{D}(j, y)$ 's toward $0 \sim 6 \mathrm{~h} \mathrm{LT}$ in the period of January March in 1996 (cf. Fig. 6). In order to confirm the existence of these anomalies and also to explore their origin, a full investigation of the solar diurnal variations at almost all the neutron monitor stations was carried out in the period 1965 2007. As a result, it has been found that these anomalies are caused by the seasonal variation of $\mathbf{F}(y)_{\mathrm{TSO}}$, which is the expression of $\mathbf{F}(y)_{\mathrm{T}}$ in solar geographic coordinates. Although $\mathbf{F}(y)_{\text {TSO }}$ is not always clearly observable by the disturbance of $\mathbf{F}(y)_{\text {DC }}$, its yearly average $\mathbf{F}_{\text {TSO }}$ can be observed almost every year, being superposed on $\mathbf{F}_{\mathrm{DC}}$. The separation between $\mathbf{F}_{\mathrm{DC}}$ and $\mathbf{F}_{\mathrm{TSO}}$, including also $\mathbf{F}_{\mathrm{HSO}}$, is, however, very difficult for the following reason. The space distributions of these fluxes which produce the first harmonic term are expressed by the spherical harmonics in the geographic polar coordinates $(r, \theta, \varphi)$, as

$$
\begin{aligned}
& \mathrm{F}_{\mathrm{DC}}=\eta_{1}(p)_{\mathrm{DC}} P_{1}^{1}\left(\cos \theta_{\mathrm{DC}}\right) P_{1}^{1}(\cos \theta) \cos \left(\varphi-\varphi_{\mathrm{DC}}\right), \\
& \mathrm{F}_{\mathrm{TSO}}=\sum_{n=1}^{2} \eta_{n}(p)_{\mathrm{TSO}} P_{n}^{1}\left(\cos \theta_{\mathrm{TSO}}\right) P_{n}^{1}(\cos \theta) \cos \left(\varphi-\varphi_{\mathrm{TSO}}\right), \\
& \mathrm{F}_{\mathrm{HSO}}=\sum_{n=1}^{2} \eta_{n}(p)_{\mathrm{HSO}} P_{n}^{1}\left(\cos \theta_{\mathrm{HSO}}\right) P_{n}^{1}(\cos \theta) \cos \left(\varphi-\varphi_{\mathrm{HSO}}\right),
\end{aligned}
$$

where $\eta(p)_{Z}, \theta_{Z}$, and $\varphi_{Z}$ with a suffix $Z$ express respectively the magnitude and the direction of $\mathrm{F}_{Z} . \mathrm{F}_{\mathrm{TSO}}$ and $\mathrm{F}_{\mathrm{HSO}}$ require at least up to the second order spherical function as they are sharply concentrated fluxes. These equations contain 16 unknown parameters. For the determination of these parameters, the observed diurnal variations shown in Fig. 3 would not be enough owing to the lack of the observations in both low latitudes and the southern hemisphere.

Even if the separation would be successful, the obtained $\mathbf{F}_{\mathrm{TSO}}$ and $\mathbf{F}_{\mathrm{HSO}}$ could not provide any information on the sidereal anisotropies $\left(\mathbf{F}_{\mathrm{T}}\right.$ and $\left.\mathbf{F}_{\mathrm{H}}\right)$ responsible for them, because they can be produced by any sidereal anisotropies from arbitrary right ascensions $\left(\alpha_{\mathrm{S}}\right)$ as long as they are subject to the seasonal variation with their maximum and minimum at the times when the Earth is closest to and farthest from their sources, respectively, as has been pointed out in Introduction. Their responsible origins can be inferred only from $\mathbf{D}(j, y)_{\mathrm{TSO}}$ and $\mathbf{D}(j, y)_{\mathrm{HSO}}$ superposed on $\mathbf{D}(j, y)_{\mathrm{DC}}$. As has been pointed out previously, the seasonal variation of $\mathbf{D}(j, y)_{\text {TSO }}$ indicates that $\mathbf{F}(y)_{\mathrm{T}}$ is maximum near the December solstice $(\alpha=6 \mathrm{~h})$, with a sharply concentrated flux from the southern hemisphere $\delta=-24^{\circ}$, while that of $\mathbf{D}(j, y)_{\mathrm{HSO}}$ is produced by $\mathbf{F}(y)_{\mathrm{H}}$, which is a maximum near the June solstice $(\alpha=18 \mathrm{~h})$ with a broad peak at the northern hemisphere $\left(\delta>0^{\circ}\right)$. As the directions of these fluxes inferred from the present analysis coincide fairly well with those obtained by the previous analyses of the sidereal diurnal variations (Nagashima et al., 1998; Nagashima and Fujii, 2006), the origin of the excess flux from $0 \mathrm{~h} \mathrm{LT}$ can be regarded as being due to the sidereal heliotail-in and helionose-in anisotropies.

Finally, it should be noted that the present conclusion does not necessarily refute the following possibility as the origin of the D-anomaly. The sidereal anisotropy which produces the required seasonal variation is not necessarily persistent throughout a year. The most extreme case of such an anisotropy is the cosmic-ray flux produced by the acceleration in a short-term period at a point on the boundary of the HMS. Such a flux is thought to be produced very frequently at any one time at any one place, as the acceleration would not always be limited only to the tail and nose boundaries, although its mechanism itself can not be directly confirmed. If such a flux happens at a point when the Earth crosses the line through the point and the Sun, it can be regarded as a kind of seasonal variation of the anisotropy required for the D-anomaly. As such happenings would be very frequently expected, their overall contribution to the D-anomaly would not be negligible.

\section{Conclusion}

The yearly averaged solar diurnal variation of cosmic rays observed with the neutron monitors and the muon telescopes on the ground does not coincide with that expected from the diffusion-convection theory. Their difference is due to the excess flux from $0 \mathrm{~h} \mathrm{LT}$ which is the yearly averaged flux in solar geographic polar coordinate, of the seasonal variation of a sidereal anisotropy having right ascension $\alpha$ with its maximum and minimum, respectively, at the times when the Earth is closest to and farthest from its source. The present analysis found two kinds of anisotropy: one shows a maximum near the December solstice $(\alpha=$ $06 \mathrm{~h}$ ), with a sharply concentrated flux from the southern hemisphere $\left(\delta=-24^{\circ}\right)$, and the other shows a maximum near the June solstice $(\alpha=18 \mathrm{~h})$, with a broad peak in the northern hemisphere $\left(\delta>0^{\circ}\right)$. The anisotropies with these directions can be identified respectively with the heliotail-in anisotropy from $\alpha=06 \mathrm{~h}$ and $\delta=-24^{\circ}$ (cf. Nagashima $e t$ al., 1998) and the helionose-in anisotropy from $\alpha=18 \mathrm{~h}$ and $\delta>0^{\circ}$ (cf. Nagashima and Fujii, 2006). Therefore, it is concluded that the departure of the yearly average of the observed solar diurnal variation of cosmic rays from that predicted by the diffusion-convection theory is due to the excess flux from $0 \mathrm{~h}$ LT produced by the seasonal variations of the heliotail-in and helionose-in anisotropies, with their maximum and minimum at the times when the Earth is closest to and farthest, respectively, from their sources.

It is noted further that the agreement of the directions of the anisotropies with those derived from the previous observations of the sidereal diurnal variations mentioned above support the previous conclusion (Nagashima et al., 1998; Nagashima and Fujii, 2006) that the major axis of the HMS in the noseward direction expected from the sidereal cosmic-ray anisotropies $\mathbf{F}_{\mathrm{T}}$ and $\mathbf{F}_{\mathrm{H}}$ is in the direction of $\alpha \sim 18 \mathrm{~h}$ and $\delta \sim 24^{\circ}$ and does not coincide with that $\left(\alpha_{\mathrm{N}}=16.8 \mathrm{~h}, \delta=-15^{\circ} \sim-17^{\circ}\right)$ inferred from the relative motion of the solar system to the neutral gas (cf. Ajello et al., 1978; McClintock et al., 1978). 
Acknowledgments. The authors express their sincere appreciation to the staffs at all the neutron monitor stations in the world and also to the staffs at the muon telescope station, the CosmicRay Research Section, Solar-Terrestrial Environment Laboratory, Nagoya Unversity, who have been responsible for the continuous long-term observations of cosmic rays.

\section{References}

Ajello, J. M., An interpretation of Mariner 10 helium (584 $\AA$ ) and hydrogen (1216 $\AA$ ) interplanetary emission observations, Astrophys. J., 222, 1068, 1978.

Bieber, J. W. and M. A. Pomerantz, A unified theory of cosmic ray diurnal variations, Geophys. Res. Lett., 10, 920, 1983

Dorman, L. I. and N. P. Milovinova, Galactic cosmic ray distribution in interplanetary space expected on the basis of anisotropic diffusion theory including the actual distribution of solar activity over solar disc, Proc. 13th Int. Cosmic Ray Conf. (Denver), 2, 713, 1973.

Earl, J. A., The effect of adiavatic focusing upon charged particle propagation in random magnetic field, Astrophys. J., 205, 900, 1976.

Fisk, L. A., Solar modulation of galactic cosmic rays, 2., J. Geophys. Res., 76, 221, 1971.

Fujii, Z., S. Sakakibara, K. Fujimoto, H. Ueno, M. Orito, and T. Yamada, Multi-directional cosmic-ray intensities, Nagoya, Report of CosmicRay Research Section, No. 18, Cosmic-Ray Research Section, SolarTerrestrial Environment Laboratory, Nagoya Univ., Nagoya, Japan, 1996.

Fujii, Z., S. Sakakibara, K. Fujimoto, M. Orito, and T. Yamada, Multidirectional cosmic-ray intensities, Nagoya, Report of Cosmic-Ray Research Section, No. 19, Cosmic-Ray Research Section, Solar-Terrestrial Environment Laboratory, Nagoya Univ., Nagoya, Japan, 2000.

Fujimoto, K., A. Inoue, K. Murakami, and K. Nagashima, Coupling coefficients of cosmic ray daily variations for meson telescopes, Report of Cosmic-Ray Research Laboratory, No. 9, Cosmic-Ray Research Laboratory, Nagoya Univ., Nagoya, Japan, 1984.

Garcia-Munoz, M., G. M. Mason, and J. A. Simpson, The anomalous ${ }^{4} \mathrm{He}$ component in the cosmic-ray spectrum at $\leq 50 \mathrm{MeV}$ per nucleon during 1972-1974, Astrophys. J., 202, 265, 1975.

Gleeson, L. J., The equations describing the cosmic-ray gas in the interplanetary region, Planet. Space Sci., 17, 31, 1969

Gleeson, L. J. and W. I. Axford, Cosmic rays in the interplanetary medium, Astrophys. J., 149, L115, 1967.

Inoue, A., M. Wada, and I. Kondo, Asymptotic direction in 1975, Cosmic Ray Table No. 1, WDC-C2 for Cosmic Rays, Institute of Phys. and Chem. Res. Tokyo, 1983.

Jacklyn, R. M., Evidence for a two-way sidereal anisotropy in charged primary cosmic radiation, Nature, 211, 690-693, 1966.

Jacklyn, R. M., Galactic cosmic ray anisotropies in the energy range $10^{11}$ $\sim 10^{14} \mathrm{eV}$, Proc. Astron. Soc. Aust., 6, 425, 1986.

Jokipii, J. R. and J. M. Davila, Effects of particle drift on the transport of cosmic rays. IV-More realistic diffusion coefficients, Astrophys. J., 248, 1156, 1981

Jokipii, J. R. and D. A. Kopriva, Effects of particle drift in the transport of cosmic rays, III Numerical methods of galactic cosmic-ray modulation, Astrophys. J., 234, 384, 1979.
Kota, J., On the second spherical harmonics of the cosmic ray angular distribution, J. Phys., A8, 1349, 1975.

McClintock, W., R. C. Henry, J. L. Linsky, and H. W. Moos, Ultraviolet observations cool stars, VII, Local interstellar hydrogen and deuterium Lyman-alpha, Astrophys. J., 225, 465, 1978.

McDonald, F. B., N. Lal, J. H. Trainor, M. A. I. Van Hollebeke, and W. R. Webber, Observations of galactic cosmic-ray energy spectra between 1 and 9 a.u., Astrophys. J., 216, 930, 1977.

Moraal, H. and L. J. Gleeson, Three-dimensional models of the galactic cosmic-ray modulation, Proc. 14th Int. Cosmic Ray Conf. (Munchen), 12, 4189, 1975.

Moraal, H., L. J. Gleeson, and G. M. Webb, Effects of charged particle drifts on the modulation of the intensity of galactic cosmic rays, Proc. 16th Int. Cosmic Ray Conf. (Kyoto), 3, 1, 1979.

Munakata, K. and K. Nagashima, A theory of cosmic ray anisotropies of solar origin, Planet. Space Sci., 34, 99-1116, 1986.

Nagashima, K. and Z. Fujii, Coexistence of cosmic-ray sidereal anisotropies originating in galactic space and at the heliomagnetospheric nose and tail boundaries, observed with muon detectors in the energy range of $60 \sim 100 \mathrm{GeV}$, Earth Planets Space, 58, 1487-1498, 2006.

Nagashima, K., R. Tatsuoka, and K. Munakata, Dependence of cosmic ray solar daily variation (1st, 2nd and 3rd) on heliomagnetic polarity reversals, Planet. Space Sci., 34, 469-482, 1986.

Nagashima, K., K. Fuijimoto, and R. M. Jacklyn, The excess influx of galactic cosmic rays from the tail of the heliomagnetiosphere, inferred from their sidereal daily variation, in Proc. of International MiniConference on Solar Particles Physics and Cosmic Ray Modulation, 93, Solar-Terrestrial Environment Lab., Nagoya Univ., Nagoya, Japan, 1995.

Nagashima, K., K. Fujimoto, and R. M. Jacklyn, Galactic and Heliotail-in anisotropies of cosmic rays as the origin of sidereal daily variation in the energy range $<10^{4} \mathrm{GeV}$, J. Geophys. Res., 103(A8), 17429-17440, 1998.

Nagashima, K., Z. Fujii, and K. Munakata, Solar modulation of galactic and heliotail-in anisotropies of cosmic rays at Sakashita underground station $(320 \sim 650 \mathrm{GeV})$, Earth Planets Space, 56, 479-483, 2004.

Nagashima, K., I. Kondo, and Z. Fujii, Sharply concentrated cosmic-ray excess fluxes from heliomagnetospheric nose and tail boundaries observed with neutron monitors on the ground, Earth Planets Space, 57, 1083-1091, 2005.

Parker, E. N., Cosmic-ray modulation by solar wind, Phys. Rev., 110, 1445, 1958.

Sekido, Y., K. Nagashima, I. Kondo, H. Ueno, K. Fujimoto, and Z. Fujii, Multi-directional cosmic-ray intensities, Nagoya, Report of Cosmic-Ray Research Laboratory, No. 1, Cosmic-Ray Research Laboratory, Nagoya Univ., Nagoya, Japan, 1975.

Yasue, S., S. Mori, S. Sakakibara, and K. Nagashima, Coupling coefficients of cosmic ray daily variations for neutron monitor stations, $R e$ port of Cosmic-Ray Research Laboratory, No. 7, Cosmic-Ray Research Laboratory, Nagoya Univ., Nagoya, Japan, 1982.

K. Nagashima, I. Kondo, I. Morishita (e-mail: isao@alice.asahi-u. ac.jp), and R. M. Jacklyn 